\title{
NASA Flight Planning Branch Space Shuttle Lessons Learned
}

\author{
Jennifer D. Clevenger ${ }^{1}$, Douglas J. Bristol ${ }^{2}$, Gregory R. Whitney ${ }^{3}$, Mark R. Blanton ${ }^{4}$ \\ NASA/Johnson Space Center, Houston, Texas 77058 \\ F. Fisher Reynolds, III $^{5}$ \\ United Space Alliance, LLC, Houston, Texas 77058
}

\begin{abstract}
Planning products and procedures that allowed the mission Flight Control Teams and the Astronaut crews to plan, train and fly every Space Shuttle mission were developed by the Flight Planning Branch at the NASA Johnson Space Center in Houston, Texas. As the Space Shuttle Program came to a close, lessons learned were collected from each phase of the successful execution of these Space Shuttle missions. Specific examples of how roles and responsibilities of console positions that develop the crew and vehicle attitude timelines have been analyzed and will be discussed. Additionally, the relationships and procedural hurdles experienced through international collaboration have molded operations. These facets will be explored and related to current and future operations with the International Space Station and future vehicles. Along with these important aspects, the evolution of technology and continual improvement of data transfer tools between the Space Shuttle and ground team has also defined specific lessons used in improving the control team's effectiveness. Methodologies to communicate and transmit messages, images, and files from the Mission Control Center to the Orbiter evolved over several years. These lessons were vital in shaping the effectiveness of safe and successful mission planning and have been applied to current mission planning work in addition to being incorporated into future space flight planning. The critical lessons from all aspects of previous plan, train, and fly phases of Space Shuttle flight missions are not only documented in this paper, but are also discussed regarding how they pertain to changes in process and consideration for future space flight planning.
\end{abstract}

\section{Introduction}

$\mathrm{T}$

HE Space Shuttle was in operation for more than 30 years. Originally conceived in part to construct a space station, delays in space station plans allowed National Aeronautics and Space Administration (NASA) to find other ways to utilize the Orbiter. Before the International Space Station (ISS) assembly missions started, Space Shuttles were tasked to perform science missions which included microgravity experiments, health and biological science, manufacturing experiments, and mapping of the surface of the Earth. The Space Shuttles deployed a number of satellites and also performed servicing and repairs of several satellites. Prior to the construction of the ISS, the Orbiter visited the Russian MIR Space Station, delivering supplies and practicing operations that would be needed for the ISS.

Throughout these flights, Space Shuttle mission planners looked for lessons that would be taken forward to make subsequent missions more efficient operationally and better for the Orbiter crews. Lessons generally focused on how

${ }^{1}$ Flight Activities Officer, Operations Division, Mail Code DO4, NASA/Johnson Space Center, 2101 NASA Parkway, Houston, TX 77058.

${ }^{2}$ Flight Activities Officer, Operations Division, Mail Code DO4, NASA/Johnson Space Center, 2101 NASA Parkway, Houston, TX 77058

${ }^{3}$ Flight Activities Officer, Operations Division, Mail Code DO4, NASA/Johnson Space Center, 2101 NASA Parkway, Houston, TX 77058.

${ }^{4}$ Flight Activities Officer, and Attitude and Pointing Officer, Operations Division, Mail Code DO4, NASA/Johnson Space Center, 2101 NASA Parkway, Houston, TX 77058, AIAA Senior Member.

${ }^{5}$ Flight Activities Officer, Operations Division, Mail Code DO4, NASA/Johnson Space Center, 2101 NASA Parkway, Houston, TX 77058. 
the Space Shuttle crew and ground controllers operated the missions as well as lessons gathered from technological upgrades to the Orbiter and ground systems. These lessons would come from internal planning debriefs, post flight crew briefings, comments from other Space Shuttle operations disciplines, and from the program offices. Some lessons were generic and affected all types of missions. Other lessons were more specific, and linked to a certain type of mission.

Here at the end of the Space Shuttle Program, mission planners have reviewed the lessons learned from the Space Shuttle missions and have placed the top critical lessons into five topics to be reviewed in detail. These lessons have been essential in developing mission plans that ensured the safety of Orbiter crews and the successful completion of mission objectives.

\section{Space Shuttle Mission Requirements}

Throughout the 30-year Space Shuttle Program, the Flight Planning branch improved its understanding of the importance of scheduling constraints and mission priorities. By defining basic guidelines and creating generic activity databases, a much more efficient and precise method of creating mission timelines emerged. Shuttle Crew Scheduling Constraints, planning Ground Rules and Constraints, Mission Priorities, and payload constraints, were tools and documents the Flight Activities Officer (FAO) used to develop each mission Flight Plan, which contained the information required for the crew and ground teams to successfully execute a mission. The history of the creation, evolution, and use of each of these documents and tools provide invaluable lessons to future spaceflight programs.

\section{A. Shuttle Crew Scheduling Constraints}

The Shuttle Crew Scheduling Constraints (SCSC) document defined the planning constraints required to maintain the crew's health and safety during Space Shuttle missions. As missions were developed, the SCSC was used by the FAO to lay down the groundwork for the crew sleep/wake cycles, based on launch and landing times. The SCSC was also used to ensure the crew was not overworked as mission objectives were assessed, and that all requirements for meals, exercise, off duty, and other basic needs were met. The SCSC document was an iterative, evolving document which started out vague and was not always adhered to, but ended up becoming a very important tool in mission planning.

Prior to the first Space Shuttle flight, the Space Transportation System (STS) Work Day Handbook was published. The stated purpose of this document was to "describe the various types of STS work days that can be used as guidelines in developing timelines for Shuttle flights." ${ }^{\prime 1}$ The document gave very broad guidelines for "typical" work days. It focused mainly on crew day length and sleep cycles, including allowances for a "typical" 20hour launch day. Over the first few years of the Space Shuttle Program, however, it was realized that these guidelines were resulting in crew fatigue, which could have led to potential errors during critical operations. Several official NASA memos were written to address these concerns, and to further define crew day length, exercise requirements, and other operational constraints.

Ultimately, the Work Day Handbook and memos were more of a collection of ideas, and not a clearly defined document. By the end of 1986 this changed. Though not as a direct result of Challenger, the grounding of the Space Shuttle fleet following the accident allowed time for the creation of Crew Scheduling Constraints, documented in Appendix K of the Space Shuttle Crew Procedures Management Plan. The purpose of the Crew Scheduling Constraints shifted away from how to develop timelines, and instead focused on ensuring the health and safety of the crew while performing tasks. The document further expanded the workday guidelines, and incorporated workload studies, circadian rhythm research, and crew comments. Appendix K defined minimum and/or maximum durations and frequency of required activities, including Post Sleep and Presleep, Meals, Exercise, Extravehicular Activity (EVA), Sleep, and crew day length. Unfortunately, although the crew constraints were established, violations of the constraints were abundant. Over $50 \%$ of the missions that occurred during the Appendix $\mathrm{K}$ timeframe processed approved exemption requests for crew scheduling constraints.

Appendix K eventually evolved into a standalone document, titled Shuttle Crew Scheduling Constraints. The SCSC continued to improve on the constraints, and the data supporting them, and included a new section specific to ISS flights. Unfortunately, NASA also continued to accept violations of those constraints, processing SCSC exception requests for 21 out of 21 missions prior to and including STS-107, the final Columbia mission.

After the Columbia accident, the Columbia Accident Investigative Board (CAIB) identified that NASA was poor at implementing policy. ${ }^{2}$ More focus was placed on all NASA policies, including the SCSC, to make sure the rules NASA set up to ensure the safety of the Orbiter and crew were being followed. Exceptions to the SCSC were still 
allowed, but only after much scrutiny at higher levels of management, and acceptance that the risk trade was weighed appropriately between the violations, the accommodations given to the crew, and mission success. In contrast to the era before the Columbia accident, only 7 missions of 22 accepted SCSC violations between the Return-to-Flight (RTF) mission, STS-114, and the final Space Shuttle mission, STS-135.

\section{B. Ground Rules and Constraints}

After the FAO developed the outline of the mission timeline, the Ground Rules and Constraints (GR\&C) was used to populate the Flight Plan with a baseline of generic activities. The GR\&C contained a list of essential activities used in various phases of a Space Shuttle mission, regardless of flight-specific objectives. These activities included Orbiter maintenance tasks, Space Shuttle robotics tasks, frequently used Orbiter attitude maneuvers, etc. The GR\&C database included activity durations, procedure details, and any scheduling or spatial constraints that dictate task order or avoidance requirements.

The GR\&C was an excellent resource for the FAO to quickly build and develop the initial timelines of a mission. The FAO did not need to research past missions, or seek answers from other Flight Control Team (FCT) members for procedures or step numbers for tasks performed regularly on each flight. The FAO avoided reinventing the wheel each time a new flight was manifested, easily compiled the needed tasks to keep the Orbiter running safely, and inserted templates for generic phases like Rendezvous, landing, or EVAs. This permitted the focus to be placed on assessing flight-specific mission objectives based on how much crew time was available after all the generic activities were implemented in the Flight Plan.

Originally, the GR\&C was a table of activities embedded in a Microsoft Word document. As more information was added to the table, the GR\&C was transitioned to a web-based tool available to both activity owners and planners. This tool allowed for better maintenance and communication of the constraints. Users always had access to the latest and greatest information. This minimized the use of incorrect information due to referencing an outdated hard copy of the document. The web-based GR\&C also provided easier search and filter capabilities of the activities. This minimized the time needed to find an activity, and reduced the chances of omitting a required system activity from the timeline. Furthermore, as changes to constraints or activity details were agreed upon, updates were made immediately without the time lag involved with editing and publishing a hard copy.

\section{Mission Priorities}

The Mission Priorities Flight Rule was a documented list of mission-specific tasks to be scheduled in the Flight Plan. The list, as the title of the rule clearly stated, was arranged in priority order, with the activity with the greatest importance to the Space Shuttle and/or ISS Program listed first. Once the basic template of the Space Shuttle mission was completed, the FAO drew from this Flight Rule to schedule as many of the big picture tasks as the available crew time allowed. It is important to note, however, that tasks were not necessarily scheduled in priority order. Due to scheduling constraints, spatial conflicts, or crew training, mission Flight Plans were built to maximize crew time and efficiency. Occasionally, this resulted in lower priority objectives occurring prior to higher priority tasks.

The Mission Priorities Flight Rule was also used to resolve conflict when crew time was not available for all the requested activities. As the crew progressed through their training, assumptions on activity durations tended to need adjustment. Sometimes, tasks took less time than originally expected, which allowed the FAO to assess additional activities from the Mission Priorities list for implementation. Unfortunately, sometimes training proved that tasks took longer than planned. The FAO used the Flight Rule to determine which tasks would be removed from the timeline or rescheduled to accommodate the necessary duration increase, or if the task that increased in time was itself the one removed from the flight.

The Mission Priorities list was not only used for preflight planning. During real-time operations, the FAO referred to this list continuously when either responding to contingencies or assessing additional flight days in the event of a mission extension. If a contingency arose during the day and crew time was spent troubleshooting, the FAO used the priorities to determine how the remainder of the mission should be scheduled. Conversely, when additional time opened up, or the decision was made to extend the mission duration by one or more days, the FAO drew from the Get Ahead Task List within the Mission Priorities to schedule more activities.

\section{Payload Constraints}

Embedded in the Mission Priorities list was the list of payloads, or experiments, to be performed during the Space Shuttle mission. Because the specific payloads differed from mission to mission, the scheduling constraints could not necessarily be transferred from flight to flight. Therefore, the constraints for each particular payload were captured in various payload-specific documents and not in the GR\&C. 
The Flight Test and Supplementary Objectives Document (FTSOD) was a collection of NASA developed vehicle and medical test objectives to be performed on one or more Space Shuttle flights. These Development Test Objectives (DTO), Detailed Supplementary Objectives (DSO), Short Duration Bioastronautics Investigations (SDBI), and National Laboratory Pathfinder (NLP) Investigations tested everything from Solid Rocket Booster performance and next-generation navigation systems, to the effects of spaceflight on sleep and the research of potential vaccines for the prevention of infections on Earth. The FTSOD contained not only the hypothesis and experiment details of each test, but also the planning constraints used by the FAO to schedule each performance of the task.

For non-ISS missions, the payload constraints were captured in the Annex 2, Part 2 of the Payload Integration Plan (PIP). The PIP was a high level document which contained the basic information and requirements of individual payloads, including Orbiter interface details, experiment weights, power levels, etc. The specific planning constraints, including attitude requirements, explicit timing or duration of tasks, etc., were housed in the Annex 2, Part 2. These constraints were used by the FAO to ensure the requirements were met, and as many of the highly desired extra objectives were assessed and scheduled.

Payload planning requirements for payloads that operated during the joint Space Shuttle/ISS missions were captured in the Earth-to-Orbit Vehicle (ETOV) Payload Activity Summary (EPAS). Based on inputs from the Payload Developers (PD), and compiled by the Marshall Space Flight Center (MSFC) in Huntsville, Alabama, the document housed all the constraints for multiple experiments, instead of focusing on just one particular payload. The EPAS covered payloads and transfer items that were conducted by both Space Shuttle and ISS crews.

Though there were multiple documents the FAO drew from to obtain the scheduling constraints for payloads, as the years went by and Space Shuttle missions became more complex, instead of listing their actual constraints the PDs began adjusting their requirements based on what they expected from the Flight Plan. By attempting to schedule the payloads themselves, the PDs unknowingly limited the amount of science a particular payload achieved. However, through multiple discussions and face-to-face meetings, the FAO repeatedly fought to learn the planning constraints in order to maximize the data achieved by each experiment.

As an example, on STS-95, the FAO worked closely with the payload customer to develop and establish good, solid requirements for the International Extreme Ultraviolet Hitchhiker - 3 (IEH-3). Also manifested on the mission was the Solar Constant (SOLCON) experiment, which had conflicting Orbiter attitude constraints with IEH-3. By helping the PD write specific requirements, instead of receiving explicit direction to schedule X here, the FAO was able to integrate the timeline optimally. Through the use of Display Electronic Unit (DEU) Equivalent loads to execute attitude maneuvers from the ground during crew sleep, IEH-3 accomplished $188 \%$ of its science requirement, and SOLCON earned $125 \%$ mission success.

\section{E. Conclusion and Lessons for the Future}

The Flight Planning Branch, and the Space Shuttle Program as a whole, grew exponentially in its understanding of the importance of scheduling constraints and mission priorities as the number of flights, and the complexity of the missions, increased. Many lessons can be drawn from the hurdles jumped along the way in order to ensure that future human spaceflight missions are as successful, if not more so, than the previous programs.

It is extremely important that crew scheduling constraints are defined upfront. They should be agreed upon by the crew, the medical community, the FCT, and the planning community to ensure not only the health and safety of the crew, but also the feasibility of meeting the constraints. Likewise, mission priorities must be documented; otherwise, chaos ensues and valuable time may be lost when crucial decisions are needed to determine which activities or payloads fit in the Flight Plan and which ones do not.

As was defined by the CAIB report, NASA and the future programs must also enforce the agreements made. ${ }^{2}$ There will be exceptions to the rules, but they should be thoroughly understood and agreed upon by all affected parties and management, and the benefits must outweigh the risks. As future programs mature, the agreements and constraints should be continuously evaluated, much like the SCSC was updated with more appropriate constraints after realizing the differences between ISS-based missions and Space Shuttle free flight missions.

Finally, the Flight Planning branch learned that the future programs and the payload community must define the actual requirements and scheduling constraints, instead of unintentionally limiting tasks based on assumptions regarding what is believed to fit in the mission timeline. The FAO should be able to utilize the defined constraints to maximize science and data output. It is unknown if more science and research could have been completed in the past if the payload community had provided the actual constraints instead of limited expectations. 


\section{Mission Plan Integration}

The process of integrating the various mission requirements into a Flight Plan evolved over the course of the Space Shuttle Program with each different type of mission flown. The planning integration challenges of Spacelab and SPACEHAB science missions were different from the challenges experienced with planning Hubble servicing, Shuttle-MIR, and ISS assembly flights; however, as the Flight Planning Branch continued to address new and novel ways to integrate diverse mission requirements into a single Flight Plan, several key concepts stood out as "lessons learned." Although future vehicles may not fly missions as complex and intricate as past Space Shuttle missions, the planning integration lessons learned will prove useful to future programs. Spaceflight will continue to be an expensive endeavor, and responsible managers will always desire to accomplish as much as possible.

\section{A. Spacelab and SPACEHAB Missions}

Many of the Space Shuttle missions flew reusable, pressurized modules which served as portable laboratories within the Space Shuttle Payload Bay. The Spacelab and SPACEHAB research modules allowed a large number of payloads to be flown on a single science-focused mission. In order to integrate the different, and at times conflicting, science objectives into the timeline and maximize the science return, the FAO worked closely with the planning specialists at the Marshall Space Flight Center (MSFC) and the PDs. It was extremely important that the planners understood not only the payload customers' requirements, but also the rationale behind the scheduling requests. Although requirements documents provided one level of communication between the science community and the FAOs, often the documents did not provide all of the information required by the FAOs to enable the maximization of science utilization. Therefore, numerous discussions between the planning teams were necessary.

Typically, the payload planners worked with the science community to develop the activities related to a particular experiment, defining the associated temporal constraints, attitude requirements, and hardware requirements. The tasks were incorporated into a Payload Crew Activity Plan (PCAP) by the payload planners and delivered to the FAO for integration into the rest of the timeline. The FAO then determined how to integrate the experiments into the timeline while balancing the needs of each payload with the Space Shuttle systems requirements and crew workload.

It was not uncommon for several obstacles to be encountered, such as conflicting attitude requirements or lack of available crew time. Occasionally, the requirements defined for the payload did not provide enough flexibility to schedule the payload. When this occurred, a series of negotiations was held between the different stakeholders to obtain a better understanding of the hard constraints and the desirements, and which could be relaxed or eliminated, without sacrificing the objectives of the science payload. Teleconferences were conducted, and for more complex problems, face-to-face meetings were set up to bring together all involved parties.

\section{B. Hubble Space Telescope Missions}

After the initial Hubble Space Telescope (HST) deploy mission, the remaining five flights to Hubble were focused on servicing the legendary telescope. Although there were a handful of middeck payloads flown on the Space Shuttle, the main objective was to complete the myriad of EVA tasks required to leave Hubble in a good posture for continued exploration. The integration effort was, therefore, less focused on accommodating many different experiments into a single mission, and instead focused on balancing HST's requirements with those of the Space Shuttle and the crew. The HST support team built upon the lessons learned from previous missions and established a good working relationship between the Goddard Space Flight Center (GSFC) and Johnson Space Center (JSC) planners, which equipped the planning teams to handle the unique challenges of each successive Hubble mission.

Each HST servicing mission included either three or five EVAs to accomplish all necessary tasks. When coupled with the high altitude of the telescope, the added risk of flying in a denser debris field, and the sensitivity of the telescope's internal components to thermal extremes, the integration challenges were often based on a combination of limits driven by either Orbiter propellant, Orbiter/HST attitude, or crew workload. What allowed the efficient integration of a complex and highly choreographed mission, was the availability of well documented payload requirements with associated rationale, and well documented agreements between each center.

Unlike the science missions where the payload activities were incorporated directly into the Flight Plan, the telescope functioned independently from the Orbiter, possessing its own line of communication with the ground for command and telemetry. To maintain the nominal operations of Hubble, the HST planners developed the telescope's Servicing Mission Integrated Timeline (SMIT) in parallel with the FAO's Flight Plan. The SMIT allowed the HST support team to schedule the commands required to safe the telescope prior to the EVAs, and verify that the work performed by the astronaut crew was a success. Timing was extremely important. GSFC and JSC worked together 
preflight to create a planning process so the two plans could be integrated with relatively few conflicts, and a process for resolving conflicts when they did arise.

Being able to have an organizational structure that permitted each stakeholder to have the opportunity to express their concerns, approval, or dissent about a particular position was also extremely important to enable each mission to have a high probability of success. Although not isolated to HST missions, Flight Technique Panels (FTPs) were held to discuss the planning process and other topics that had an impact on the entire HST and Orbiter team. With regard to plan integration, a FTP was the forum in which multidisciplinary conflicts were resolved or elevated to a higher authoritative body when consensus could not be obtained at lower levels. The availability of a single defined authority was very important to ensure that the process of integrating a mission plan continued to move in a positive direction and did not result in wasteful gridlock.

\section{Shuttle-MIR Missions (Phase I)}

The Phase I Program was the predecessor to the ISS Program. The concept of using the Space Shuttle to build a space station was not new, and NASA had spent years working with the European, Canadian, and Japanese space agencies developing operational concepts and hardware. The Russians, on the other hand, had years of practical hands-on experience with assembling and operating numerous space stations. In the early 1990s, the two major superpowers, who had developed their own unique way of operating, were challenged with the task of bringing the Space Shuttle and MIR Space Station together. The underlying goals of the Phase I Program were to pave the way for two former adversaries to find common ground, and to establish the foundation on which all future cooperative efforts between the two nations would be built. The Shuttle-MIR Program was the first step toward a multi-decade, unified effort to complete the most complicated and expensive project in human history.

The planning integration challenges that arose with the Shuttle-MIR Program were born, in part, from differences in language, culture, and philosophy. However, the most challenging aspect to enabling the NASA and Russian planning teams from truly integrating a mission plan was the pervasive idea that each side's way of doing business was the right way. It became apparent that face-to-face meetings between the planners was not adequate, and that personnel from NASA would need to have a permanent presence in Moscow to work side-by-side with their Russian counterparts during each mission. Not only was having embedded personnel invaluable to smoothing out real-time communication between each control center, but each side had the opportunity to see firsthand how the other side really operated. NASA and Russian planners learned from each other and gained an appreciation and understanding of why the two sides had different approaches to similar problems.

Integrating the Russian and NASA Flight Plans was a slow process, and it was important for the FAOs to allow plenty of time to build the Flight Plan. The Flight Plan was written in both Russian and English, and time was required for interpreters to translate the data being exchanged between the two control centers. Negotiations also took longer, and sometimes simple issues were difficult to resolve because a key point was lost in translation, delayed by time zone differences, or simply received in a completely different way than was intended. Planning a Space Shuttle flight to MIR, although not as technically challenging as Space Shuttle science flights or HST missions, was a complicated and time consuming affair, and both sides learned to have more patience.

Perhaps more so than any previous type of Space Shuttle mission, the Shuttle-MIR missions were primarily focused on the education of the different teams involved, and the joint Russian-American team needed to adjust to the new way of doing business. The FAOs and Russian planners started out on opposite sides of the planning spectrum, but eventually found a way to reach a certain level of trust, an important milestone in planning. The key was to continue to learn about the other side, to continue to allow enough time to work through the process so that deadlines did not create additional stress, and to never underestimate the value of simple gestures of kindness and camaraderie when smoothing out disagreements.

\section{International Space Station Missions}

On December 6, 1998, the ISS became a reality when the American Unity module attached to the Russian Zarya module. At that moment, the process of integrating Flight Plans changed once again, and continued to evolve until the last flight of the Space Shuttle Program in July of 2011. As assembly of ISS pressed on, new players were added to the integration process. Activity inputs now literally came from all over the world. New ways to document changes to the plan were introduced to the teams. And all this helped define how ISS steady-state operations occur today.

The task of assembling an integrated Flight Plan for an ISS mission now not only involved Russian planners, but also another organization internal to the Flight Planning Branch: the ISS Operations Planners. The Ops Planners were the ISS planning counterparts to the Shuttle FAO, and were responsible for planning the ISS crew and vehicle systems tasks. Early flights to the ISS were choreographed mainly by the FAO team, but as the ISS grew in 
complexity and began to support a crew of its own, the lead responsibility for joint Space Shuttle/ISS missions were soon shared by the FAOs and Ops Planners. With two sibling organizations focused on the assembly of the ISS, it became apparent that the planning integration function could greatly benefit from consolidated planning systems, shared development costs to improve and create the required tools to support the consolidated systems, and collaboration on new ways to automate the physical integration of planning data.

The FAOs and Ops Planners were the lead integrators for joint mission planning, but the amount of crew tasks and ground commanding required for ISS assembly and utilization flights was considerable. To compensate for the increase in the number of activities, planning duties were distributed across five different control centers. The FAOs and Ops Planners at JSC, Russian planners in Moscow, U.S. payload planners at MSFC, planners from the European Space Agency in Munich, Germany, and Japanese planners in Tsukuba, Japan, were all involved in the development of the mission plan. Each control center was the expert of their own systems and modules, and it was very helpful to have a planning system that allowed the planners around the world to input changes to the plan for automatic data integration into a master database. The Consolidated Planning System (CPS) was developed for use as the single application to create joint Space Shuttle and ISS timelines and to facilitate the automatic integration of planning data. Interface agreements were also established to allow partner agencies to export files that could be combined in CPS for planning integration. This saved countless man-hours and reduced the number of errors caused by manual data entry.

Distributed planning also meant that plan changes needed to be coordinated among the different control centers across multiple time zones. In past Space Shuttle missions, planning primarily occurred in Mission Control CenterHouston (MCC-H) with supporting locations either in Houston or other NASA centers. During ISS missions, however, planning occurred across the globe with final integration of each agency's plans in MCC-H. Plan changes had to be coordinated with each FCT which levied the requirement for additional configuration management tools while still providing for the capability to quickly change the plan based on real-time events. The result was a combination of the traditional Space Shuttle planning coordination with the ISS steady-state planning change request process.

A Space Shuttle mission typically had a high intensity, highly optimized plan, and due to its dynamic nature was replanned each night based on previous events. On ISS, because the crew was on orbit for months at a time, and because of the high number of control teams around the world, the focus of the planning process was placed on allowing more team review and coordination time to reduce the number of late changes and risk of error. In order to merge these two planning philosophies during joint Space Shuttle/ISS missions, a replan template was formalized between NASA and the International Partners (IPs). The template specified how changes were submitted to the FAO and Ops Planner for incorporation into the Flight Plan. Early deadlines were introduced for big picture changes to the timeline to allow time for translation and coordination internal to each center. Later milestones were also documented for detailed changes so all teams were clear regarding when the final timeline was being uplinked to the crew for execution.

The necessity for a hybrid Space Shuttle/ISS planning change process was so that the ISS planning team could continue to meet their obligations to their planning counterparts in Europe, Russia, and Japan while not overburdening the Space Shuttle planning team with the additional overhead of the ISS process. In the end, the joint mission replanning process successfully implemented each partner's requirements for communicating and approving plan changes without greatly slowing down the replanning process.

\section{E. Conclusion and Lessons for the Future}

With each progressive mission, the Space Shuttle planning team learned how to continually improve ways to integrate various mission requirements into an efficient and successful sequence of events. Although the customers and objectives for each mission changed, the expectation was always the same: find a way to maximize the capabilities of the flight crew and vehicle to accomplish as much as possible. Although the future destination, objectives, and vehicle will be quite different from what was experienced during the Space Shuttle Program, the lessons learned during the 135 flights of the Space Shuttle are still applicable.

One key to successfully integrating inputs and enabling efficient coordination is to spend time early on with all of the stakeholders to establish an effective planning process. It is vital that all participants understand how plan additions and changes will be communicated, establish required milestones, and determine who will lead the integration process to minimize the confusion among planners across the world. The amount of time spent working out the details is related to the number of different groups responsible for developing the plan, so it is very important to begin the process development early on. If possible, it is extremely advantageous to run a simulation or process verification with all participants and tools that are expected to be used. Some disconnects will not become obvious until the process is tested from end to end. It also provides important practice for the control teams. 
Managers will also still expect to accomplish as much as possible during missions because spaceflight continues to be an expensive endeavor. The expensive nature of future missions means there is a high probability for joint international missions which would necessitate the need to integrate multiple agencies' requirements. It is imperative that future flight planners build upon the experience of Space Shuttle and ISS flight planners and continue to build strong relationships with the stakeholders of the mission. Open communication with each planning representative must be established, well documented requirements must exist with rationale, and effective tools that provide efficient data flow between planners should be developed. In addition to tools and processes, it is essential that a relationship based on mutual understanding, respect, and trust must exist between planners. Only with that type of working relationship can teams have the type of communication that lead to solutions rather than impasses. Spending time with other teams in face-to-face settings (Technical meetings, training sessions, after work socials) provide the opportunity for each team to build a good rapport with the other. An upfront investment in education about the operational constraints, goals, desires, organizational cultures, vocabulary, and capabilities of each team is invaluable toward building a cohesive team that is able to tackle the challenges of preflight preparation and solve problems encountered during the mission.

\section{Contingency and Alternate Timelines}

During a Space Shuttle flight, the execution of a mission could drastically change in the event of a single failure. However, the time it takes to respond to a failure could be greatly reduced with preparation and anticipation of contingency cases. Planned responses, including mission timelines, were often built preflight in order to serve as a building block during a mission, should a failure occur. Some of these contingency cases were universal to all missions, and some were strictly mission-specific. One contingency case in particular, STS-400, was planned as a full, stand-alone Space Shuttle mission. The preparation and use of these alternate timelines are invaluable lessons that should be utilized in future exploration programs.

\section{A. Universal Cases}

Prior to a mission, the organizations responsible for building a mission (i.e. Flight Director, Program Office, Engineering, Crew, Mission Operations Directorate (MOD) disciplines, etc.) determined which failures had a higher likelihood of occurring, or which failures, should they occur, had the greatest impact to the mission plan. Though the number of potential contingencies seems endless, there were a few distinct failures that could occur on any mission. Similarly, vastly different contingency cases could require similar responses, which drove a need to plan for such responses every mission.

As a Space Shuttle mission neared the end of its flight, several critical events occurred in order to prepare for safe entry. On the day prior to landing, it was typical for the crew to stow the Ku-band antenna, the Space Shuttle Remote Manipulator System (RMS), and the radiators. If any of these pieces of external equipment did not stow in its proper entry configuration, there was a risk of the Payload Bay Doors (PLBD) not closing completely, which would prohibit a 'Go' for landing. Failures such as these would require the crew to perform an EVA to manually stow or repair the broken hardware. Likewise, during the Deorbit Prep phase on the day of landing, if the PLBD did not close due to a hardware contingency, the crew would be required to manually close the doors.

For each of these cases, the crew and ground FCT would be required to react quickly to schedule an EVA. In order to minimize the time between the failure and landing, timelines were created to cover these scenarios and published in the Deorbit Prep Checklist. These generic timelines, flown on every mission, could be altered depending on when the failure occurred, what actually failed, the amount of consumables available onboard, and when the next landing opportunity was scheduled. However, the basic building blocks of the response were already laid out in order to allow the team to focus on the details of the repair, instead of spending precious time on the big picture.

Another contingency scenario universal to all flights was the Minimum Duration Flight (MDF). In an MDF case, a failure occurs which allows the mission to continue to meet the highest priority objectives of the flight, but requires the Orbiter to land as soon as possible once they are complete. This ultimately results in rapid re-planning of the entire mission with a much shorter duration.

Though the concept of the MDF was universal, the exact content of what tasks were to be performed were evaluated on a flight-specific level. Preflight, the MDF Flight Rule was written to capture the required tasks and basic timeline to be followed. The content was based on the Mission Priorities Flight Rule, fulfilling the highest priority objectives. A high level Overview Timeline was created by the FAO as a visual representation of the MDF scenario. An Overview Timeline was sufficient because it was uncertain when the failure would occur and/or if further discussion would be required. Additionally, there would be sufficient time to react and generate a more 


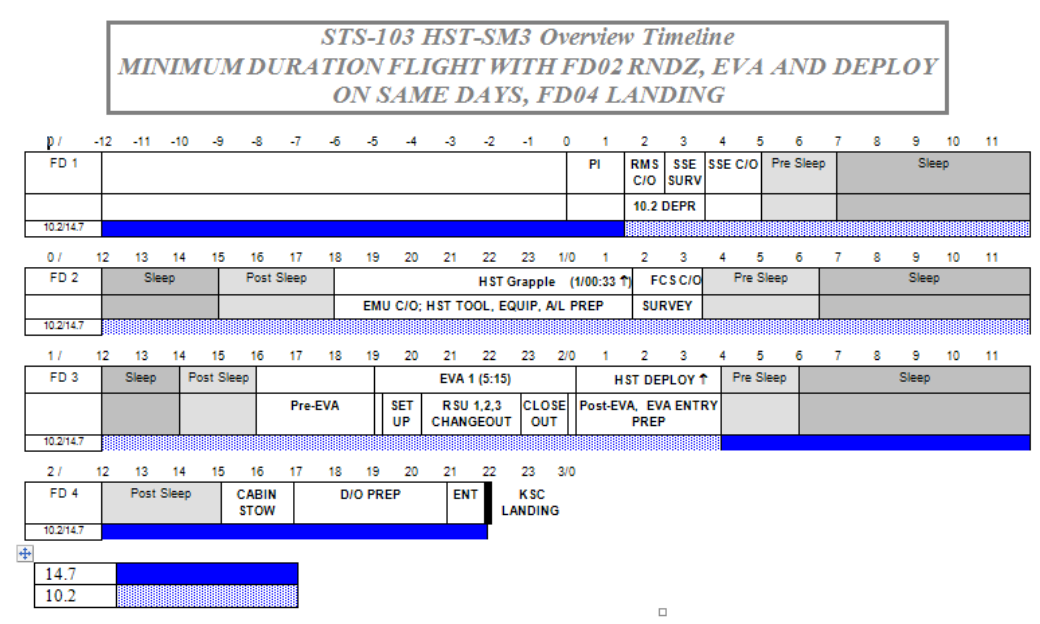

Figure 1. STS-103 Minimum Duration Flight timeline. detailed plan. The MDF timeline offered a good starting point to initiate discussions on how to react to the shortened mission. In real time, these timelines could be modified to add lower priority objectives if the Mission Management Team (MMT) deemed the risk acceptable.

MDF timelines were executed for STS-44 and STS-83. STS-44 was shortened due to the loss of one of three Orbiter Inertial Measurement Units (IMUs), but completed its major mission objectives. STS-83, however, was ultimately reflown as STS-94 due to a problem with one of the Orbiter's Fuel Cells. Figure 1 shows the MDF timeline created for STS-103, a HST

repair mission. The timeline demonstrates that the HST would be captured, one EVA would be performed to repair the highest priority HST equipment, HST would be deployed, and then the Space Shuttle would return home. Fortunately, the MDF timeline was not executed for STS-103, and the full duration mission was accomplished.

\section{B. Flight Specific Timelines}

Though there were a few universal failures or scenarios that could occur, most alternate timelines were prepared for flight specific situations. The planning team often built contingency or alternate timelines to react to these occasions so the crew and flight controllers could more efficiently react to the situation. The level of detail and effort put into these contingency timelines often varied with the likelihood of occurrence, the severity of the impact of a failure, and the time available to produce the contingency plan in real time. Payload deploys, unscheduled EVAs, delays in nominal operations, and unexpected mission extensions were common situations discussed during pre-mission planning.

Throughout the Space Shuttle Program, the planning team regularly produced contingency satellite deploy timelines. As an example, on Inertial Upper Stage (IUS) deploy missions, backup deploy timelines were published in the Flight Plan to allow immediate access to the contingency plan. Figure 2 is the one-orbit late deploy timeline for the Tracking and Data Relay Satellite (TDRS) deployed on STS-56. The greater level of detail in this timeline was required due to the time criticality and the complexity of the deploy operations. Additional contingency timelines for a two-orbit late deploy and a one-day late deploy were also published on the IUS deploy missions. Luckily, the contingency IUS timelines were not used during missions, though they were regularly used during simulations to train crewmembers and flight controllers.

EVAs also required significant planning. Prior to a mission, the FCT would determine if there were a likelihood of a quick response EVA. Unscheduled EVA timelines were

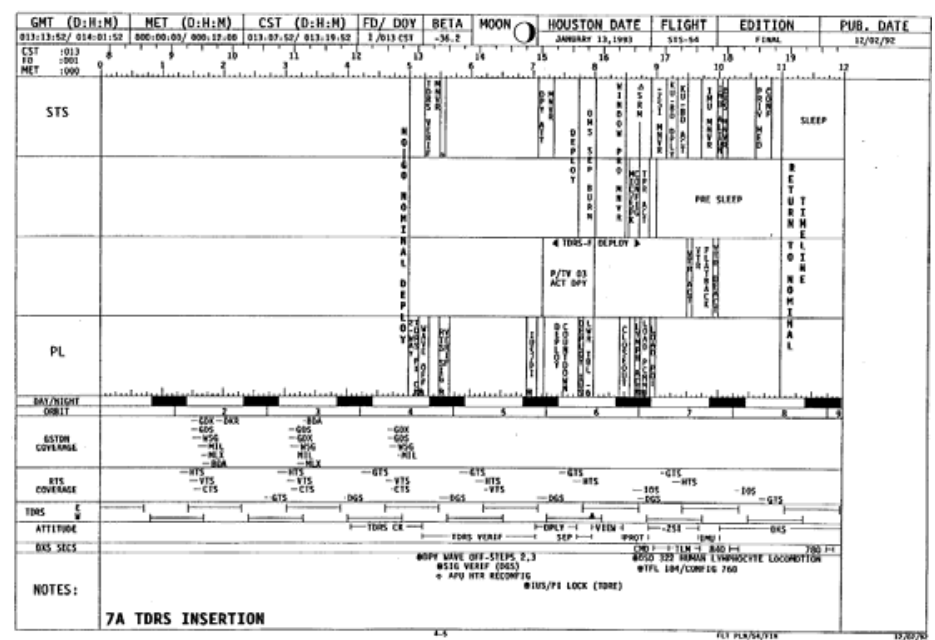

Figure 2. STS-56 one-orbit late deploy timeline. frequently developed to minimize the real-time impacts in replanning for failures that either had a high probability of occurring, or a large impact to mission success. As an example, a quick response EVA timeline was developed for STS-37, which launched the Gamma Ray Observatory (GRO). The Extravehicular (EV) crewmembers were nominally scheduled to perform many of the EVA Prep activities just short of donning the spacesuits. This would allow the crew to perform an immediate EVA in the event of a problem with the Solar Array (SA) deploy. Whereas the SA deploy on the mission was successful, the High Gain Antenna (HGA) failed to deploy when commanded. 
Since the EV crew was already prepared to egress the Orbiter, the FCT was able to easily modify the pre-mission unscheduled EVA timeline in order for the crew to manually free the HGA.

During ISS assembly missions, contingency timelines were frequently built to allow quick recovery in the scenario where the installation of a new module did not proceed as expected. For example, STS-124 had a contingency case ready in the event that the primary power string in the new Japanese laboratory did not activate nominally. Through simulations and discussions over several years, the FCT determined in advance what criteria would be used to consider the primary power channel failed, and what actions were required by the crew and ground to activate the backup power channel and safely enter the new module. The case involved a complex choreography of ground commands which could not have easily been built in real-time due to the coordination required between the NASA and Japanese flight controllers. This information was well documented in a contingency timeline that was not necessary during execution, however, would have been invaluable in the event of a failure.

Other contingency cases did not require the creation of an actual timeline; however, high level discussions were beneficial for the FCT to understand the impacts and planned responses. For example, most missions to the ISS had a backup plan in the event of a one-orbit late docking scenario. Typically, the flights to the ISS had high priority tasks, including intense robotics activities, scheduled immediately after docking. Because there was a short timeframe between docking and crew sleep, a delay in any of these activities would have a considerable impact to the remainder of the mission. Preflight discussions with the crew and the FCT were necessary to determine the most efficient and appropriate course of action, so precious time was not wasted on orbit.

Though most alternate timelines were created as a pre-emptive response to failures, other timelines were created in case extra time became available on orbit. Space Shuttle consumables were predicted preflight using conservative assumptions to support nominal mission duration. Sometimes these consumables allowed for a real-time mission extension, if it was deemed necessary. To prepare for this possibility, alternate timelines were often developed prior to a mission to preplan the extra days. In real-time, when it was determined that Space Shuttle consumables would allow for a mission extension, the MMT directed the FCT to incorporate the additional days into the timeline. If the alternate timeline was developed preflight, the timeline was transitioned seamlessly with little extra work. This philosophy was used frequently later in the Space Shuttle Program and especially for the joint Space Shuttle/ISS missions. The alternate timelines streamlined review of the timeline, and limited discussions about requirements and priorities in real-time, therefore allowing the planning team and flight controllers to focus on other aspects of the mission.

\section{Contingency Mission - STS-400}

As a result of the loss of Columbia during the landing attempt of STS-107, the CAIB recommended NASA research all options for crew survival in the event of an emergency. Referred to as a Launch On Need (LON) flight, a means of rescuing the Space Shuttle crew on an alternate vehicle was planned in case the Space Shuttle's Thermal Protection System (TPS) could not be cleared or repaired for entry, or if there were a critical failure preventing the Space Shuttle from safe entry.

STS-125, the final HST repair mission, was a unique case regarding the rescue flight requirement. Unlike ISS missions, where the Space Shuttle crew could utilize the ISS as a safe haven until another Orbiter could be readied, the STS-125 crew would have limited resources and time to wait for a rescue vehicle. To minimize this wait time, a second Space Shuttle sat on the launch pad ready to launch within 3-7 days of being required. Labeled STS-400, this LON flight was planned like every other mission, with a detailed Flight Plan that included launch, rendezvous with Atlantis, transfer of the HST crew, and a safe return of Endeavour, the second Orbiter. Like several of the previously mentioned contingency cases, STS-400 also went through various simulations and training scenarios with the crew and ground FCT to ensure the preparedness of NASA for this extremely complex mission.

\section{Conclusion and Lessons for the Future}

Contingency planning was a steady force over the years of the Space Shuttle Program, and it evolved and changed with each mission. Though the types of alternate plans, from simple discussions to full mission timelines, varied from flight to flight, many of the underlying characteristics provide excellent lessons learned that can be utilized by future spaceflight programs.

Contingency planning can be time consuming, and the number of potential failures unending, but every minute spent preflight paves the way for a faster turnaround time in-flight. It is important to avoid battling too many failures, and direction must be taken to focus on those failures that are more probable, or have the largest impact to mission success. However, any big picture upper level decisions that are made ahead of time result in efficient and well thought out timelines and procedures. This maximizes precious onboard crew time in the event of a system or hardware failure. 
In situations where the contingency and alternate timelines were used, planning was significantly more efficient than those times where unforeseen problems occurred and the crew and FCT had to react. However, another significant contribution from the alternate timelines came when similar, unexpected situations occurred. In the case of STS-37, though the anticipated failure of the Solar Array deploy did not happen, the pre-mission planning laid the foundation to be able to react to a comparable situation. Flexibility is an extremely important tool when responding to contingencies.

Once the alternate timelines were constructed, the plans were regularly used in mission simulations. Practicing off-nominal, more difficult timelines make real-time execution of the nominal timeline seem effortless. The more training the FCT and crew receive for a particular failure, the less chaos ensues when the failure actually presents itself. The team remains calm under pressure, and an organized effective plan is executed.

Future manned space programs would do well to take the additional time to work contingency scenarios prior to a mission. Whereas it is impossible to develop a timeline for every situation or even predict every scenario, lessons learned from planning multiple scenarios will prepare crewmembers and flight controllers for many situations that might occur. Although most of the contingency planning performed pre-mission will not be used, it frequently initiates valuable discussions that allow the crew and FCT to further learn the mission objectives and develop a cohesive team. As new endeavors in human spaceflight are pursued, new obstacles will be created. It is important for future programs to remember that preparation can mean the difference between failure and success.

\section{Planning Tools}

During the Space Shuttle era, internal software applications were developed by NASA which allowed the FAOs to create a set of timelines for a Space Shuttle flight. These timelines, combined to create the mission Flight Plan, were used by the Space Shuttle crews to carry out the objectives of each flight. Once created, a timeline could be easily altered as preflight requirements changed or real-time anomalies arose. As technology advanced, and Space Shuttle missions increased in complexity, the software applications evolved to meet the needs of the FAO. Several lessons can be drawn from the history of the Space Shuttle planning tools and utilized by future programs to minimize complications in the early stages of development.

\section{A. Software Applications}

The first application developed was the Crew Activity Planning System (CAPS). CAPS required users to view information on two large computer monitors. One monitor was used to display digital data associated with each activity scheduled in the mission. Planning engineers manually entered activity information, including the name, duration, and procedure checklist references into the system. These activities were then scheduled on the appropriate crewmembers. The second monitor was used to display the scheduled activities in a graphical timeline in a "what you see is what you get (WYSIWYG)" format. The orientation and positioning of the text associated with each scheduled activity on the monitor represented how the timeline would look as a hardcopy printout used by the crew and FCT during Space Shuttle flights. The system allowed the scheduled activities to be displayed in two different graphical formats. One format, referred to as the Summary Timeline, used a twelve hour timescale per page which allowed a single day's schedule to be viewed on two pages. The Summary Timeline gave a view of each Flight Day's activities to view task durations and order. The second format, referred to as the Detail Timeline, used a four hour timescale per page which provided space for detailed crew instructions and checklist references.

After several years of use, CAPS evolved into CAPS II, which used newer technology to simplify the hardware configuration (e.g., one monitor was used for both graphical and digital information). This later evolved into the Flight Planning System (FPS) which improved on CAPS and CAPS II with hardware and software design updates. In addition to manual scheduling capabilities, FPS now had the ability to import timeline information for Spacelab flights that was generated by the payload planning engineers at the MSFC.

As planning tools were being developed for support of ISS, the decision was made to create a single software application to be used by both Shuttle and ISS planners. The Consolidated Planning System (CPS) was created to merge some of the capabilities of FPS with those being developed for the ISS. Like its predecessors, CPS used advances in hardware and programming languages to improve the planning process. Whereas the project management for the CAPS, CAPS II and FPS had been housed within the planning organization, the project management for CPS was placed into a different organization. Requirements for CPS were documented and meetings were held to allow users to provide recommendations to the developers. However, because the designers were not planners, initially there was a lack of understanding of how user interaction with the system should be constructed for the tool to be practical. Some of the early deliveries of CPS did not meet the expectations of the 
planning engineers, and the software had to go through several updates before it could be used to support a Space Shuttle flight. Eventually, the project management of the system moved back into the planning organization.

During early CPS development, user requirements to obtain digital data from the system were deferred to the later years due to schedule and budget concerns. Digital data such as activity names, task start and end times, and scheduled crewmembers had been available with FPS, and planners found it very useful in analyzing a timeline. For example, the data could be extracted from FPS and sorted and filtered to confirm all expected activities were scheduled or SCSC constraints were met. It could also be distributed to outside organizations for high level management information, including the start times of high-profile mission objectives. Since the capability to retrieve the data from the planning system was not on the horizon, users created in-house scripts to pull the desired data from the CPS database. These scripts used a graphical user interface to retrieve the data and provided output in various formats. Macros were also created, which simplified the loading of these data into spreadsheets for analysis.

\section{B. Conclusions and Lessons for the Future}

Since the beginning of the Space Shuttle Program, planning tools played an integral part in the role of the FAO. Timelines to be used by the crew and ground teams were vital in preflight mission development and simulations. The capability to easily update these timelines both preflight and in real-time was crucial to establish and maintain the most efficient use of the crew's time while onboard the Space Shuttle. Future programs should continue to invest in software which allows planners to easily plan and replan the activities needed for a flight.

Including the users in the design of the system is also extremely important to ensure that all requirements and preferences are addressed. It is essential that the user interface is suited to the operator's needs. When creating a tool development schedule, time should be provided up front for the early design of the user interface. Too much focus on only the functionality of an application will result in a piece of software that is not practical. To aid in the development, and eventual upgrades, the project management for the application should remain close to the ultimate users. Having the developers close to the users provides a more frequent and efficient dialog which will provide a useful application from the beginning. The development of any large scale application should also not preclude the development of user scripts that can be used to augment the capabilities of the tool.

\section{Spacecraft Versatility and Flight Planning}

Throughout the Space Shuttle Program, FAOs and Attitude and Pointing Officers were repeatedly able to utilize the flexibility and versatility of the Orbiter. The Space Shuttle had the capability to re-orientate itself to complete mission objectives. It also carried multiple payloads that posed constraints on the Orbiter mission, had a communication system that was used efficiently with attitude changes, and an evolutionary approach to the uplink and downlink capabilities of crew data. The adaptability and evolution of the Space Shuttle and her systems provide lessons that should be seriously considered in the development of future programs.

\section{A. Thermal Capabilities and Capacity}

One of the harshest environments that any spacecraft must endure is space itself. Extreme temperature swings between sunlight and deep space tend to dictate a specific spacecraft orientation in order to maintain the integrity of hardware. The Space Shuttle is no different from other spacecraft, however, the capability and flexibility to handle changes to orientation is much broader. Throughout the program, operational needs of the spacecraft itself were defined, problems occurred, and payloads dictated attitude requirements which drove the need to expand the operational thermal envelope.

Operational considerations were made to accommodate the Orbiter in various phases of flight. As an example, a series of attitude maneuvers was required to prepare the Orbiter for Earth entry and landing. The IMUs, which provide information for use in the navigation system, were aligned via two star trackers several hours before the first deorbit opportunity. The Orbiter maneuvered to two different attitudes that aligned a pair of stars, one star in each tracker. These attitude maneuvers and the operations of aligning the IMUs were required to be completed approximately four hours prior to the deorbit burn. Once the IMUs were aligned, the Orbiter was maneuvered to a radiator cold-soak attitude at exactly four hours prior to the burn. Two-hours and fifteen minutes prior to the deorbit burn, the Payload Bay Doors were finally closed. Another attitude was then used to keep the cabin and Payload Bay cooler, as well as provide for near-continuous communications with the Mission Control Center.

Occasionally, problems arose that required attitudes beyond the nominally scheduled thermal envelope. On STS4, a unique thermal problem occurred when "some rainwater penetrated protective coating of several tiles while the Orbiter was on pad. On orbit, the affected area was turned toward sun and water vaporized, preventing further tile damage from freezing water." Additionally, on STS-8, "The nose of Orbiter was held away from the sun for 14 
hours to test the flight deck area in extreme cold." ${ }^{4}$ This type of ingenuity, using the Orbiter's attitude to solve a system concern, was incredibly important in finding the limits of the system.

Some payloads possessed thermal requirements that either increased science output or kept hardware from breaking. The role of the Attitude and Pointing Officer was to ensure these requirements were met, while also upholding the constraints of the Orbiter itself. It was a balancing act that many times tested the limits of both payload and Orbiter.

Payload customers were required to meet certain Space Shuttle thermal constraints to be considered for the manifest on a mission. In addition to flying in a benign Payload Bay pointed to Earth attitude, the experiment hardware had to be able to withstand a certain amount of time in specially defined attitudes. These attitudes included, but were not limited to, the Orbiter Boom Sensor System (OBSS) survey attitudes, the series of ISS rendezvous burn attitudes, and attitudes to condition the main landing gear tires prior to landing.

SOLCON, flown on STS-95, was one of these payloads with requirements that conflicted with Orbiter constraints. For mission success, SOLCON required its hardware, flown in the Payload Bay, to be pointed to the Sun for a specific amount of time. The Space Shuttle, however, had a documented constraint that limited the amount of sunlight in the PLB, as it caused Orbiter hardware to get too warm. These Space Shuttle requirements and capabilities were document in the Flight Rules to provide the operators with the absolute criteria used in the evaluation of the spacecraft and the expected recovery times for orientations that could have an effect on the system. Ultimately, the Attitude and Pointing Officer was able to create an Attitude Timeline that allowed SOLCON to obtain more than its expected science while still maintaining the safety and integrity of the Orbiter.

Additionally, each mission was screened by thermal engineers to verify the Attitude Timeline accounted for all of the constraints and did not stretch beyond operational boundaries. If new operations were to be performed, the engineers worked in concert with the Attitude and Pointing Officers to develop techniques for bringing the spacecraft back within the envelope following the new operations. This relationship helped in increasing the thermal envelope.

\section{B. The Unique Challenges of Mission Payloads and Mission Objectives}

Working with payload customers often provided interesting challenges for the Attitude and Pointing office. In addition to thermal constraints, each payload brought unique integration challenges to the flight. Likewise, mission objectives sometimes contributed to the trials of merging various constraints and requirements.

Deploying payloads from the Space Shuttle contributed to the difficulty of incorporating multiple requirements and constraints for the Attitude and Pointing office. On STS-41B, the Pointing Officer had to design an attitude to protect the Orbiter windows from the Payload Assist Module (PAM), attached to the PALAPA and WESTAR satellites as they were deployed from the Payload Bay. In addition to safeguarding the Orbiter, the Space Shuttle was required to quickly maneuver to provide tracking of the satellites after they were released. An example of the attitude sequences is set forth below:

1) The Orbiter will be in a -Z-axis Local Vertical spacecraft protective attitude when entering the pre-deploy activities. $^{5}$

2) Following the Orbital Maneuvering System (OMS) separation burn, the Orbiter will return to a Local Vertical Local Horizontal (LVLH) attitude to allow for visual tracking of the payload. ${ }^{5}$

3) Prior to the PAM perigee burn the Orbiter will maneuver to an attitude that will protect the windows from erosion by the PAM exhaust plume. This places the Orbiter nose-forward with the Payload Bay away from the Earth. The PAM will be ahead of and below the Orbiter with the Orbiter pitched up $\sim 50$ degrees to their common line-of-sight. ${ }^{5}$

Occasionally, payloads used the ability to change the Space Shuttle's orientation to provide improved lighting conditions. On STS-41D a "102-foot-tall, 13-foot-wide Office of Application and Space Technology (OAST-1) solar wing extended from the Payload Bay. The wing carried different types of solar cells and extended to its full height several times. It demonstrated large lightweight solar arrays for a future in building large facilities in space such as a space station." ${ }^{6}$ The solar wing had to be illuminated by changing the Orbiter attitude while still meeting the mission constraints, as in Fig. 3.

STS-114, the first flight following the Columbia accident, brought a new set of

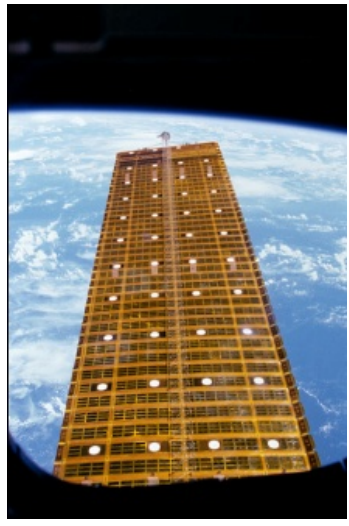

Figure 3. Solar Array Wing deployed during STS-41D. NASA imagery. constraints with the CAIB's recommendation for inspection of the TPS. The OBSS was a new piece of hardware flown in the Payload Bay and used to inspect the TPS. During the second crew day on 
orbit, the astronauts used the Space Shuttle RMS to pick up the OBSS and maneuver a sensor package attached to the end of the OBSS to various spots along the wings, nose, and crew cabin, as seen in Fig. 4.

The sensors mounted on the OBSS had multiple field-of-view constraints. The Sun could not be placed directly behind the sensor as it would cause shadows. The Sun could not be in front of nor face the camera as it would blind the sensor. The OBSS also could not be radiated by the Orbiter's Ku-band antenna which was used to downlink all of the real-time images and playback video of the inspection. By using established techniques to build blockage

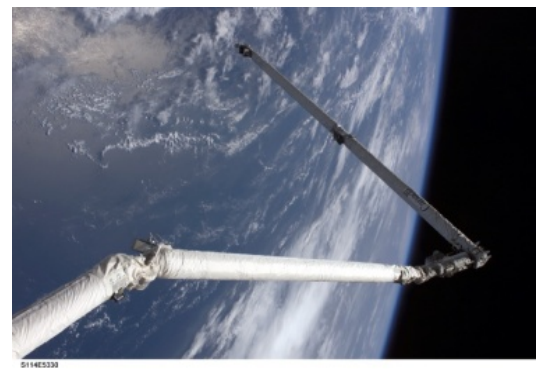

Figure 4. Orbiter Boom Sensor System attached to the Shuttle Remote Manipulator System. NASA imagery. diagrams, much like when solving the Ku-band masking issue, sensor fields-of-view were created and modeled. Attitudes were ultimately designed to protect the sensor suite during the TPS inspection.

For STS-125, the final HST servicing mission, the Attitude and Pointing office was again tested. First, the mission was planned at a higher altitude than typical missions. This higher altitude contains more orbital debris which must be avoided. Secondly, since a rescue mission was required for the mission, onboard consumables had to last until STS-400 arrived. An attitude plan was devised to minimize maneuvers to save propellant and to minimize the potential for critical impacts to the Orbiter's TPS.

Further complicating the STS-125 attitude plan were the EVAs scheduled during the mission. Critical hardware and scientific instruments were moved from their storage containers in the Orbiter's Payload Bay to Hubble to replace or upgrade equipment. As the hardware was transferred, however, no sunlight could be present on the crewmembers, hardware, or the open bays of Hubble. A complicated Attitude Timeline was built around all of these constraints, and despite the challenges, STS-125 was an extremely successful mission.

A final example of a unique mission requirement was the fly-around photography during the STS-134 mission. Whereas the primary mission objective of installing the Alpha Magnetic Spectrometer (AMS) was successful, attitude planning and coordination with the Russian space agency, Roskosmos, provided everyone with one of the most iconic images of the Space Shuttle Program. Pointing Officers worked with requirements from the Roskosmos and the ISS Program to develop an attitude sequence and Solar Array configuration to meet power and thermal requirements of both the Space Shuttle and ISS vehicles. The Space Shuttle was used to maneuver the entire ISS complex as the Russian Soyuz capsule undocked to prepare for landing. As the Soyuz backed away from the vehicles, fantastic photos of the Space Shuttle docked to the ISS were taken for the first time. Without the ingenuity and out-of-the-box thinking of the Attitude and Pointing office, this view would never have been seen.

\section{Communications Line-of-Sight}

As technology improved, the availability of a high data rate antenna meant planners and the STS Program could downlink live television of public affairs events, science experiment data, and external video. STS-8 brought additional testing of the operational environment and began a phase of high data rate communication. "Testing was

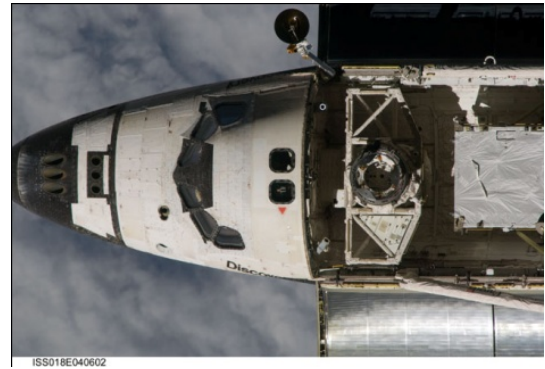

Figure 5. STS-119 Rendezvous Pitch Maneuver photography from the ISS. NASA imagery.

the sky, as seen in Fig. 6.

With an obstructed view of the sky, scheduling events that required high data rate Ku-band signals sometimes required changes to the Orbiter attitude. The Attitude and Pointing Officer worked with the FAO to determine if the event was able to be rescheduled over a good line-of-sight between the Ku-band antenna and the TDRS. If the timeline dictated specific timing of an event, then the Attitude conducted between the Tracking and Data Relay Satellite-I (TDRS-1) and the Orbiter using a Ku-band antenna."F The Orbiter Ku-band dish, when deployed from the Payload Bay, extended past the starboard side outside of the sill. The antenna had both a "wrist" and an "elbow". The elbow was fixed at full deploy or retract, and the wrist operated in two axes. The Kuband dish can be seen at the top of the Fig. 5, just above the crew cabin and to the left of the starboard Payload Bay doors and radiator. Though the Ku-band dish could move and track the TDRS for better line-of-sight, this particular deploy position did not provide an unobstructed view of 14 
Timeline was evaluated for changes to the Orbiter's orientation to bring in a solid line-of-sight. It was very important, however, that the new attitude still meet the constraints of the Space Shuttle and any payloads in the Payload Bay.

During the early years of the program, the FCT was able to protect the crew and Orbiter hardware via a mask. These masks were two-dimensional boxes described by the azimuth and elevation angle from the zero-zero position of the Ku-band antenna reference frame, and via a "wrist" angle or beta angle. When the antenna's line-of-sight

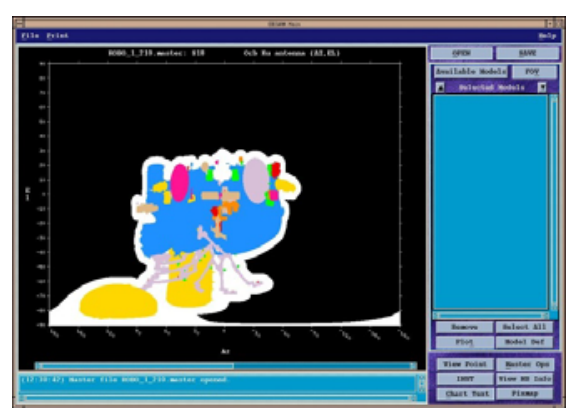

Figure 7. STS-135 MPLM position plot. A plot of the ISS (blue), MPLM (yellow), and SSRMS (gray) sequence of positions during installation procedure. entered into the bounds of the mask, the antenna stopped radiating, though continued to track the TDRS. As the line-of-sight tracked past the bounds of the mask, the antenna began sending the signal again.

Later in the Space Shuttle Program, it was determined that supplementary software needed to be added on the Orbiter to protect payloads and optimize Ku-band coverage. Using up to four additional software masks, the restricted area could be further refined so that those zones would not be radiated with the Ku-band beam. Software was developed that helped in the optimization of these protection boxes. For example, the new software allowed for modeling of the motion of hardware on the end of the Space Shuttle RMS. Figure 7 represents the model of the ISS and the installation procedure of the Multipurpose Logistics Module (MPLM) on STS-135. The MPLM, ISS, and Space Station RMS are plotted for the installation procedure. Once the motion was plotted, the boxes were developed to provide protection for all of the hardware visible to the Ku-band antenna in addition to using the nominal beta line and Orbiter mask.

\section{Data Uplink and Downlink}

From previous experience with manned spaceflight, it was clear that a means to uplink new procedures and schedules to the Space Shuttle crew would be needed. Several uplink methods were developed, some more reliable than others, to meet the need to deliver these updates to the crew. As the Space Shuttle Program matured, personal laptop computers were certified to fly in the Orbiter and a method to access these laptops from the ground was developed. This provided a mechanism to both uplink procedures more quickly and to downlink data files.

As Space Shuttle flights began, uplink of messages such as procedures and timeline updates, was performed using a teleprinter. Ground personnel manually created a message, typing the required text into a very early text editor. The message was limited to a subset of ASCII characters (e.g. all capital letters, a handful of special characters) and saved to a floppy disk. When a communications link was established with the Orbiter, either through a ground station or later through the NASA TDRS system, the message was transmitted directly to the teleprinter as a series of audible tones on one of two audio channels normally used for crew communication. Though this system was useful in expanding communication with the crew, there were complications. If a communication dropout occurred in the middle of the uplink, the portion of the message being transmitted at that time was lost and a retransmission of the message might be needed. Additionally, the uplink rate was limited to $32 \mathrm{kbps}$.

Once the early TDRS satellites were in their final locations, an alternate means to uplink messages became available. The Text and Graphics System (TAGS) was basically a facsimile machine onboard the Space Shuttle. Ground personnel scanned messages into a device which then transmitted the digital data through the Ku-Band system to the TAGS machine on the Orbiter. Because TAGS required the Ku-band link for uplink, it was only available when the Space Shuttle had a communications link through the TDRS system. TAGS was a great improvement over the teleprinter. Since the messages were scanned, all types of information were sent to the crew including text and graphics. The uplink rate for TAGS was $128 \mathrm{kbps}$, substantially faster than what was available for the teleprinter link. Unfortunately, the onboard TAGS machine was notorious for allowing paper to jam. Due to the design of the machine, paper jams were very difficult to clear. Once a jam occurred, it was very probable that the uplinks to TAGS would no longer be available for the remainder of the flight, and all future uplinks would need to downmode to the teleprinter.

Eventually, a more reliable onboard printer, the Thermal Impulse Printer System (TIPS), was used onboard for the uplink of messages. Ground preparation of messages was performed by manual entry into a word processor or by use of a scanner. Digital uplink of the messages to the TIPS printer used the $128 \mathrm{kbps}$ window in the Ku-Band system, as did the TAGS uplink. The message was printed on thermal paper onboard. Due to its design, the TIPS was essentially jam free. Any paper jams that did occur were easily cleared by the crew. After several successful 
flights, the teleprinter, which was still being flown as a backup, was removed from the Orbiter and a spare TIPS machine was stowed as a backup.

As commercial laptops began to fly onboard the Space Shuttle, NASA engineers developed the Orbiter Communications Adapter (OCA), a custom network card that would allow the ground to interface with the onboard laptops. The OCA connection was a two-way connection, providing both an uplink path to and a downlink path from the onboard laptops. The OCA used the same $128 \mathrm{kbps}$ window that was used by TAGS and TIPS for the uplink. It also provided a connection into the onboard Ku-Band signal processor to provide a downlink connection of either $2 \mathrm{Mbps}$ or $4 \mathrm{Mbps}$. With the OCA in place, the message uplink concept was changed from real-time onboard printing, which was used with all previous uplink methods, to uplinking files to the onboard laptops. Once onboard, these files were either printed by a ground command or viewed by the crew through a web browser. Messages to be uplinked were prepared using any commercial office application (e.g., Word, Excel, Powerpoint, etc.).

With the new downlink capability available through the OCA link, ground personnel were able to downlink and deliver various types of files created by the crew or onboard payloads, including digital imagery, system information (e.g. fuel cell data, rendezvous tools information), and crew e-mail. Following the Columbia accident, the OCA link was used to downlink various types of inspection files, including imagery and accelerometer data, which were used to assess the integrity of the Orbiter for entry. Late in the program, an updated OCA card provided an additional downlink interface into the Ku-Band's 48 Mbps channel, which greatly reduced the time required to downlink crew photographs and inspection imagery.

Finally, beginning with STS-123, the capability was added to the onboard laptops that allowed them to connect to the laptop network onboard the ISS while the Shuttle was docked. This allowed ground personnel to utilize the ISS OCA link to access the Space Shuttle laptops for uplinks and downlinks when the Space Shuttle Ku-Band was not available.

\section{E. Conclusion and Lessons for the Future}

Future spacecraft programs should look to the capabilities of the Space Shuttle. During the operational phase of the Space Shuttle Program, engineers and operators expanded the Orbiter's operational envelope in the areas of payloads, thermal, communications, and uplink and downlink capacity. The ability to adapt was an important key to the success of the program.

Developing a new spacecraft with the entire operational envelope analyzed would be cost prohibitive. To offset cost, future programs should strive from the beginning to provide as much operational flexibility as possible. The Space Shuttle program showed that over the life of the program additional analysis, mostly driven by mission or payloads requirements, made the spacecraft easier to operate and accommodate new spacecraft operations. Whether due to a system failure, a payload with new operational requirements, or the spacecraft itself, future vehicles should be versatile enough to change orientations with minimal impact to the structure.

Communication with a spacecraft is also extremely important. The Attitude and Pointing flight controllers adapted and designed software and processes to solve the line-of-sight problems associated with moving hardware relative to the eye-point, such as an antenna. It is in the best interest of future programs to explore all options, from tracking capabilities of an onboard antenna to having the means to re-orient the spacecraft itself, to improve communication coverage. Additionally, future vehicles should anticipate spacecraft body and payload blockage and have a means to protect hardware.

With any manned spacecraft, a method is also needed to provide the crew with updated information and to downlink crew generated information. These data are not the same as vehicle command and telemetry, and a separate system may be warranted to separate the vehicle command and telemetry from the operational information. The design of spacecraft communications should always be forward looking in terms of data rates available, flexibility and potential for upgrade to allow newer ground technology to take full advantage of interfacing with the onboard. A high rate uplink system should also always be required, especially for longer duration flights, to allow large files (e.g. videos, music) to be sent to the crew.

\section{Conclusion}

Space Shuttle missions have allowed mission planners to accumulate a large amount of data concerning how astronauts live and operate in space, and how ground controllers interface with the crew and each other. The capabilities of the Orbiter have provided a variety of different types of missions and operations to be planned. Lessons from these missions will be used as a starting point for creating new requirements for future human spaceflight. 
Experience has shown that preflight planning, cooperative relationships, and preparing for contingencies are well worth the time spent, and help pave the way for successful missions. Spacecraft and ground systems flexibility provide the crew and mission controllers increased options to satisfy mission priorities and any inflight issues that arise.

Future missions, whether to an asteroid, the Moon, Mars, or deeper into the Solar System, will need to leverage off the experiences of the Space Shuttle in order to create better tools for ground operations, and a more capable and adaptable spacecraft. Also, future human operations in space will require collaboration between NASA organizations, industry, academia and International Partners. The success of missions to come depends on how prepared we are for those missions before launch.

\section{Acknowledgments}

The authors of this manuscript extend sincere appreciation to the dozens of members of the Flight Planning Branch who served the Space Shuttle Program throughout the years. Without the knowledge, passion, and creativity of the planning team, the success of each mission would not have been possible. Special acknowledgment is granted to Roger Smith, Jennifer Price, Tracy Scott, Terry Clancy, and Patty Casas, who provided guidance, support, and memories which contributed greatly to this manuscript. Finally, the authors recognize Marguerite Weatherall with genuine gratitude, as without her expertise in vocabulary and grammar, this manuscript would have been published with too many commas and dangling participles to count.

\section{References}

${ }^{1}$ Freeman, D. L., “STS Work Day Handbook,” JSC-10541, 14 Feb. 1980, p.1.

${ }^{2}$ Columbia Accident Investigation Board, “Columbia Accident Investigation Board”, NASA, Aug. 2003.

${ }^{3}$ Space Shuttle Mission Archives, URL: http://www.nasa.gov/mission_pages/shuttle/shuttlemissions/archives/sts-4.html [cited 16 Aug 2011]

4 Space Shuttle Mission Archives, URL: http://www.nasa.gov/mission_pages/shuttle/shuttlemissions/archives/sts-8.html [cited 16 Aug 2011]

${ }^{5}$ Ferguson, B. E., “41-B (STS-11) Crew Activity Plan,” JSC-18840, 19 Dec. 1983.

${ }^{6}$ Space Shuttle Mission Archives, URL: http://www.nasa.gov/mission_pages/shuttle/shuttlemissions/archives/sts-41D.html [cited 16 Aug 2011] 


\section{Acronyms}

\begin{tabular}{|c|c|}
\hline AMS & Alpha Magnetic Spectrometer \\
\hline CAIB & Columbia Accident Investigation Board \\
\hline CAPS & Crew Activity Planning System \\
\hline CPS & Consolidated Planning System \\
\hline DEU & Display Electronic Unit \\
\hline DSO & Detailed Supplementary Objectives \\
\hline DTO & Development Test Objectives \\
\hline EPAS & ETOV Payload Activity Summary \\
\hline ETOV & Earth-to-Orbit Vehicle \\
\hline EV & Extravehicular \\
\hline EVA & Extravehicular Activity \\
\hline FAO & Flight Activities Officer \\
\hline FCT & Flight Control Team \\
\hline FPS & Flight Planning System \\
\hline FTP & Flight Technique Panel \\
\hline FTSOD & Flight Test and Supplementary Objectives Document \\
\hline GR\&C & Ground Rules and Constraints \\
\hline GRO & Gamma Ray Observatory \\
\hline GSFC & Goddard Space Flight Center \\
\hline HGA & High Gain Antenna \\
\hline HST & Hubble Space Telescope \\
\hline IEH-3 & International Extreme Ultraviolet Hitchhiker - 3 \\
\hline IMU & Inertial Measurement Unit \\
\hline IP & International Partner \\
\hline ISS & International Space Station \\
\hline IUS & Inertial Upper Stage \\
\hline JSC & Johnson Space Center \\
\hline LON & Launch On Need \\
\hline LVLH & Local Vertical Local Horizontal \\
\hline MCC-H & Mission Control Center-Houston \\
\hline MDF & Minimum Duration Flight \\
\hline MOD & Mission Operations Directorate \\
\hline MPLM & Multipurpose Logistics Module \\
\hline MSFC & Marshall Space Flight Center \\
\hline NASA & National Aeronautics and Space Administration \\
\hline NLP & National Laboratory Pathfinder \\
\hline OAST & Office of Application and Space Technology \\
\hline OBSS & Orbiter Boom Sensor System \\
\hline OCA & Orbiter Communications Adapter \\
\hline OMS & Orbital Maneuvering System \\
\hline PAM & Payload Assist Module \\
\hline PCAP & Payload Crew Activity Plan \\
\hline $\mathrm{PD}$ & Payload Developers \\
\hline PIP & Payload Integration Plan \\
\hline PLBD & Payload Bay Doors \\
\hline RTF & Return-to-Flight \\
\hline SCSC & Shuttle Crew Scheduling Constraints \\
\hline RMS & Remote Manipulator System \\
\hline SA & Solar Array \\
\hline SDBI & Short Duration Bioastronautics Investigations \\
\hline SMIT & Servicing Mission Integrated Timeline \\
\hline SOLCON & Solar Constant \\
\hline STS & Space Transportation System \\
\hline
\end{tabular}


TAGS

TDRS

TIPS

TPS

WYSIWYG
Text and Graphics System

Tracking and Data Relay Satellite Thermal Impulse Printer System

Thermal Protection System

What You See is What You Get 


\section{NASA Flight Planning Branch Space Shuttle Lessons Learned}

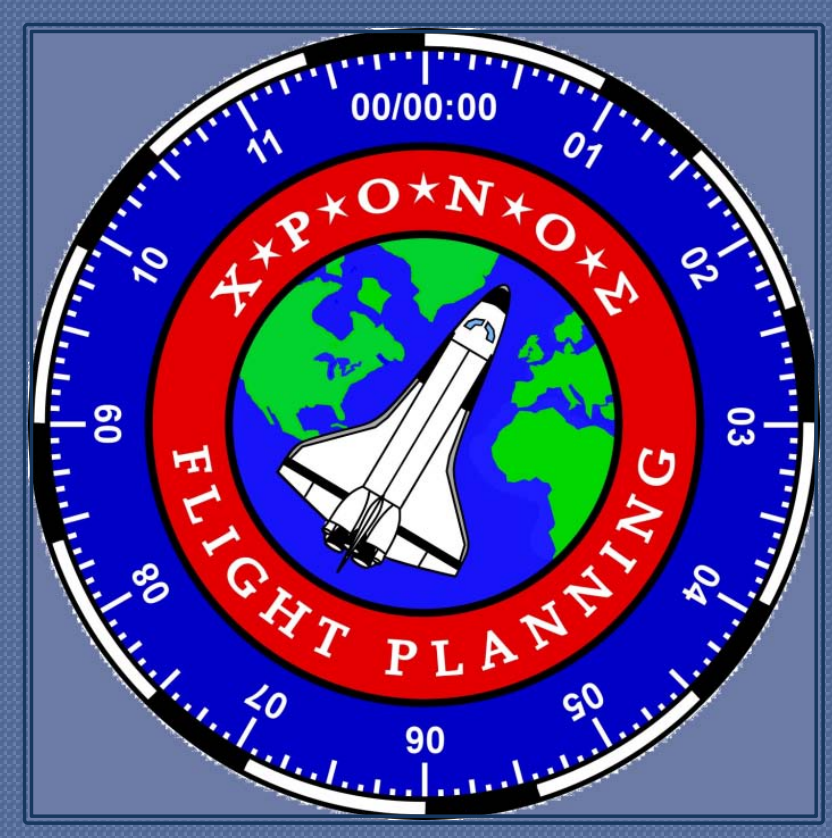

Jennifer D. Clevenger, NASA Douglas J. Bristol, NASA Gregory R. Whitney, NASA Mark R. Blanton, NASA F. Fisher Reynolds, III, USA 


\section{Execute Package Comedy}

Throughout the Space Shuttle Program, the Flight Activities Officer (FAO) compiled the daily Execute Package, containing procedure updates, new timelines, and other messages for the crew. On the front cover of the Execute Package was a bit of comedy, provided by the FAO team. It served as comic relief, and at times reflected a solemn tribute, for the Astronaut crews and the Flight Control Teams. Though not officially a lesson learned, always remember to enjoy what you do and have fun.
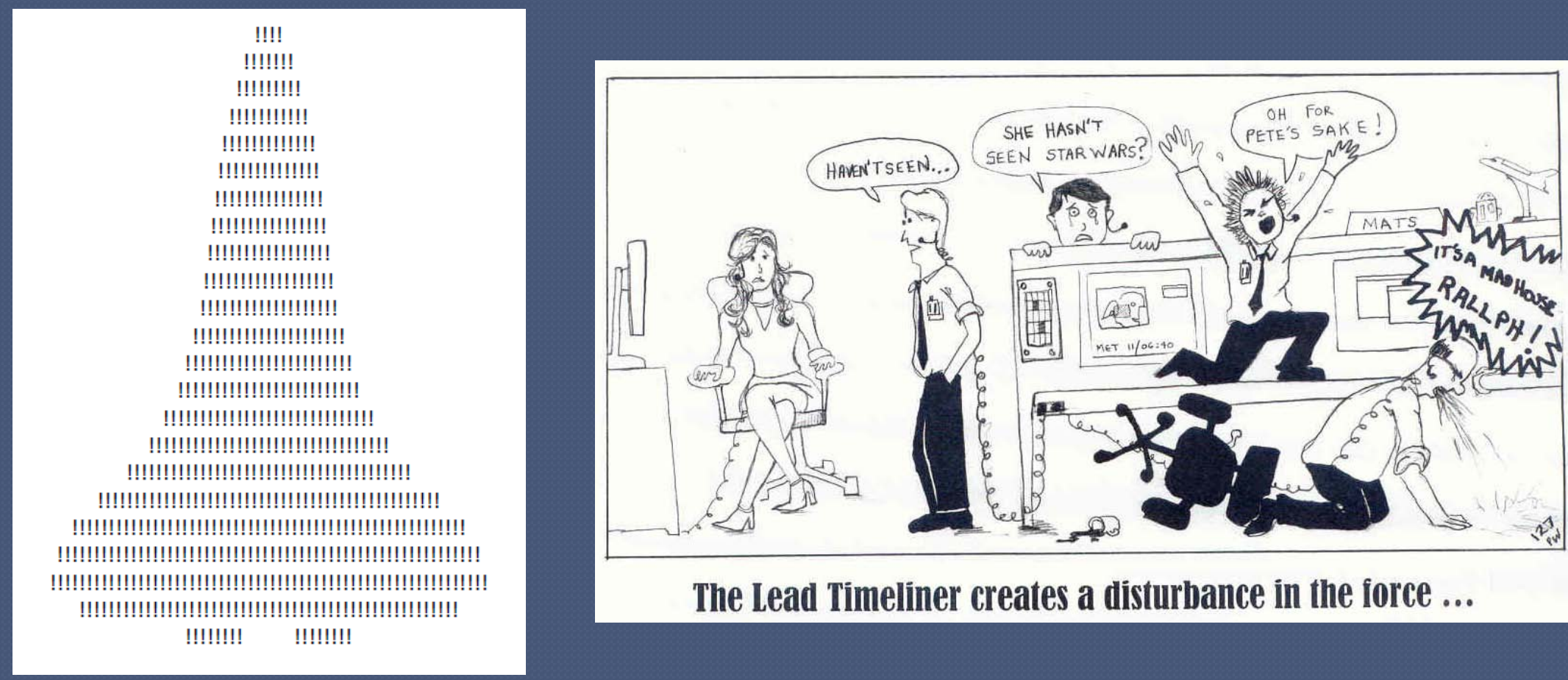

The Lead Timeliner creates a disturbance in the force ... 


\section{Introduction}

- Planning products and procedures that allowed the mission Flight Control Teams and the Astronaut crews to plan, train, and fly every Space Shuttle mission were developed by the Flight Planning Branch at the NASA Johnson Space Center in Houston, TX

- Lessons learned were collected from each phase of the Space Shuttle mission

Building the initial Flight Plans

Integrating inputs from multiple partners and payloads

Developing contingency and alternate plans

Utilizing software tools used to create and update the Flight Plans

Embracing the versatility of the Orbiter itself to maximize science, focus on safety, and enhance communication with the crew 


\section{Space Shuttle Mission Requirements}

- Several documents were used by the FAO to develop the mission timeline required to execute a Space Shuttle mission

- Shuttle Crew Scheduling Constraints

- Defined the planning constraints required to maintain the crew's health and safety

- Ground Rules and Constraints

Database used to populate the Flight Plan with a baseline of required generic activities

- Mission Priorities

Documented mission-specific tasks, with the activity with the greatest importance to the Space Shuttle and/or ISS Programs listed first

- Payload Constraints

Collection of various documents containing objectives, hardware and scheduling constraints and requirements for payloads flying on both the Space Shuttle and ISS 


\section{Example Summary Timeline - \\ STS-134}

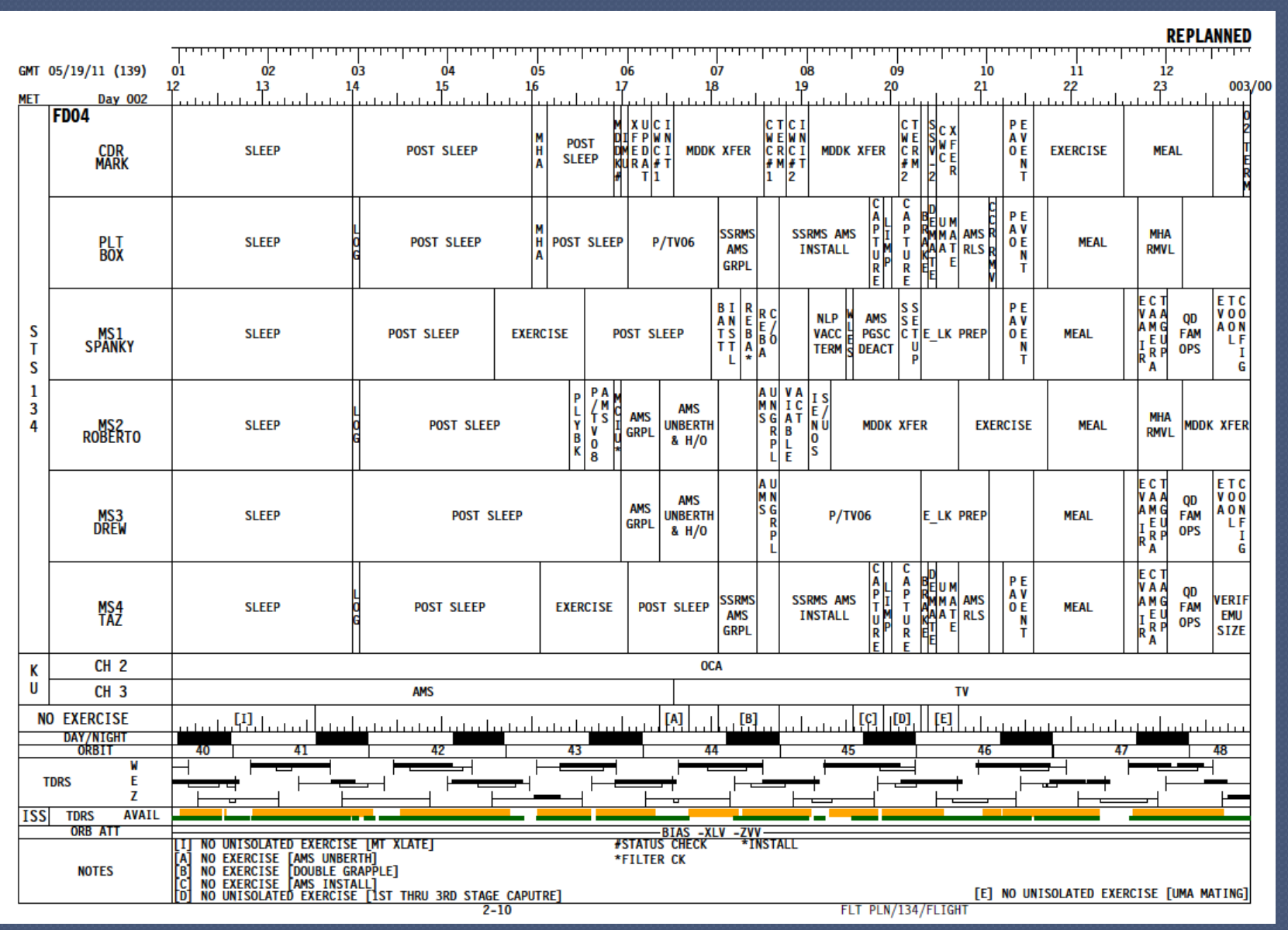

- SCSC: populated Sleep, Post Sleep, Meals, Exercise

- GR\&Cs: populated generic EVA prep tasks, IMU align, CWC fills

- Mission Priorities: AMS install was highest priority task, however, on the previous day a lower priority task, ELC-3 install, occurred

- ELC-3 was installed first to avoid a CG issue in the event of an emergency landing case

- Payload Constraints: added Sleep Logs, Viable, IENOS, NLP Vaccine tasks 


\section{Space Shuttle Mission Requirements Lessons}

- Define crew scheduling constraints upfront

- Document mission priorities to assist with initial building of the mission plan, as well as to be used in the event of a conflict

o Enforce the agreements made!

Exceptions to the rules must be documented, understood, and agreed upon by all parties

○ Define the actual requirements and constraints, instead of limiting tasks based on assumptions regarding what is believed to fit in the timeline

This will maximize the amount of science and research 


\section{Mission Plan Integration}

- Various types of Shuttle missions required completely different approaches to plan

- Spacelab/SPACEHAB science missions

Large number of payloads flown on a science-focused mission required integration of different, and at times conflicting, science objectives

- Hubble Space Telescope (HST) missions

Complex and highly choreographed missions with 3-5 EVAs, thermal and propellant constraints, and a telescope that functioned independently from the Orbiter

Required intense planning integration of the Shuttle Flight Plan and the HST Servicing Mission

Integrated Timeline (SMIT)

- Shuttle/MIR missions

Established the foundation for all future efforts between the US and Russia

Introduced new planning challenges with differences in culture, language, time zones, and approaches to spaceflight

- Shuttle/ISS missions

Introduction of Ops Plan, the ISS planning team

High distribution of planning duties across the world: FAO/Ops Plan at JSC, U.S. Payloads at Marshall Space Flight Center (MSFC), and Russian, European, and Japanese planners

Replan template was formalized with early milestones set for big picture plan changes to allow time for translation, and late milestones defined for last-minute detailed changes 


\section{Example HST Timeline - STS-125}

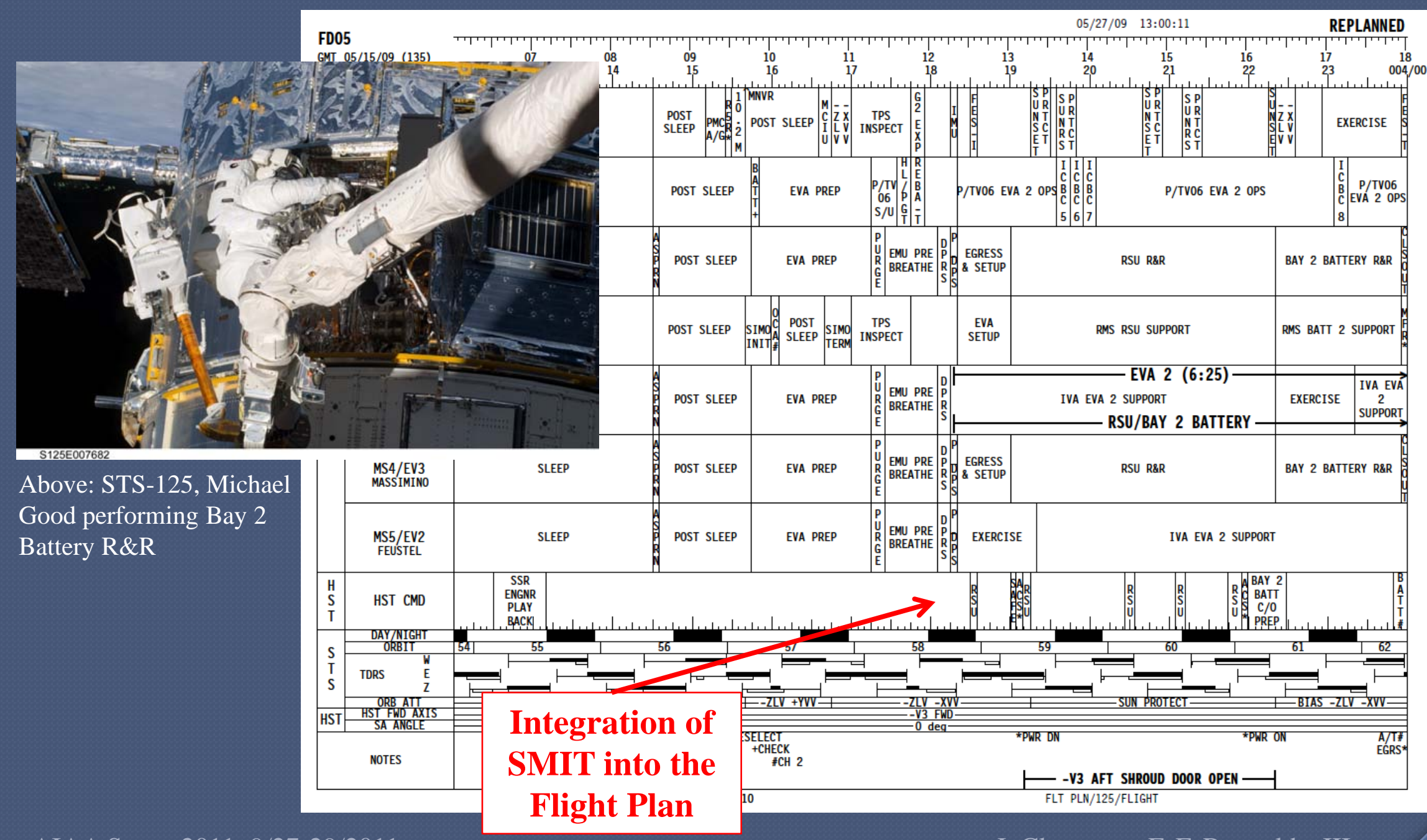




\section{Spacelab, SPACEHAB, Shuttle/MIR,}

Satellite Deploy, and ISS Missions

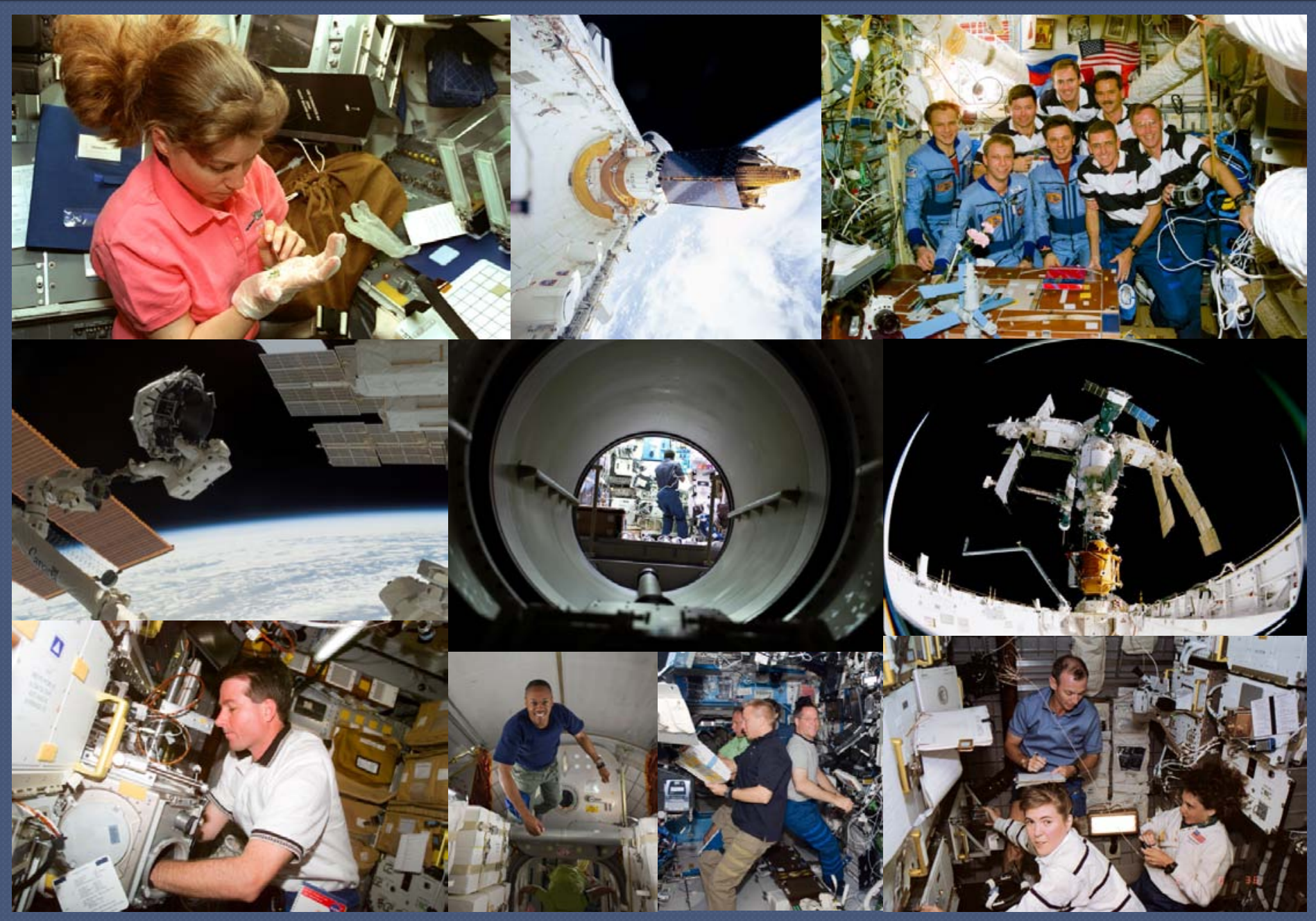

From top, clockwise: STS-93, STS-43, STS-74, STS-74, STS-57, STS-128, STS-133, STS-95, STS-118; Center: STS-107 


\section{Mission Plan Integration \\ Lessons}

- Establish an effective planning process

- Understand how plan additions and changes will be communicated, establish milestones, determine who will lead the final integration

○ Tools and processes are important, but humans must still work with humans

- Build strong relationships with all stakeholders

Face-to-face meetings provide better communication of each participants' expectations, and build trust and respect 


\section{Contingency and Alternate Timelines}

- A single failure can define a mission, but planned responses created preflight can be an invaluable tool when time is of the essence

- Universal cases

Few distinct failures that could occur on any mission were identified and led to generic plans published in the Deorbit Prep Checklist

Minimum Duration Flight allowed mission to continue to meet highest priority objectives, but land as soon as possible

- Flight Specific cases

Level of detail ranged from discussion of planned responses to publishing of actual timelines

Regularly produced contingency satellite deploy, quick response EVA, ISS assembly contingency, and mission extension timelines

(.) STS-400

- Created as a Launch-On-Need flight in the event the STS-125 Orbiter could not be cleared for safe entry, and the crew needed to be rescued

Planned as a full-up mission, ready to launch within 3-7 days of being required 


\section{Example Contingency Timelines}

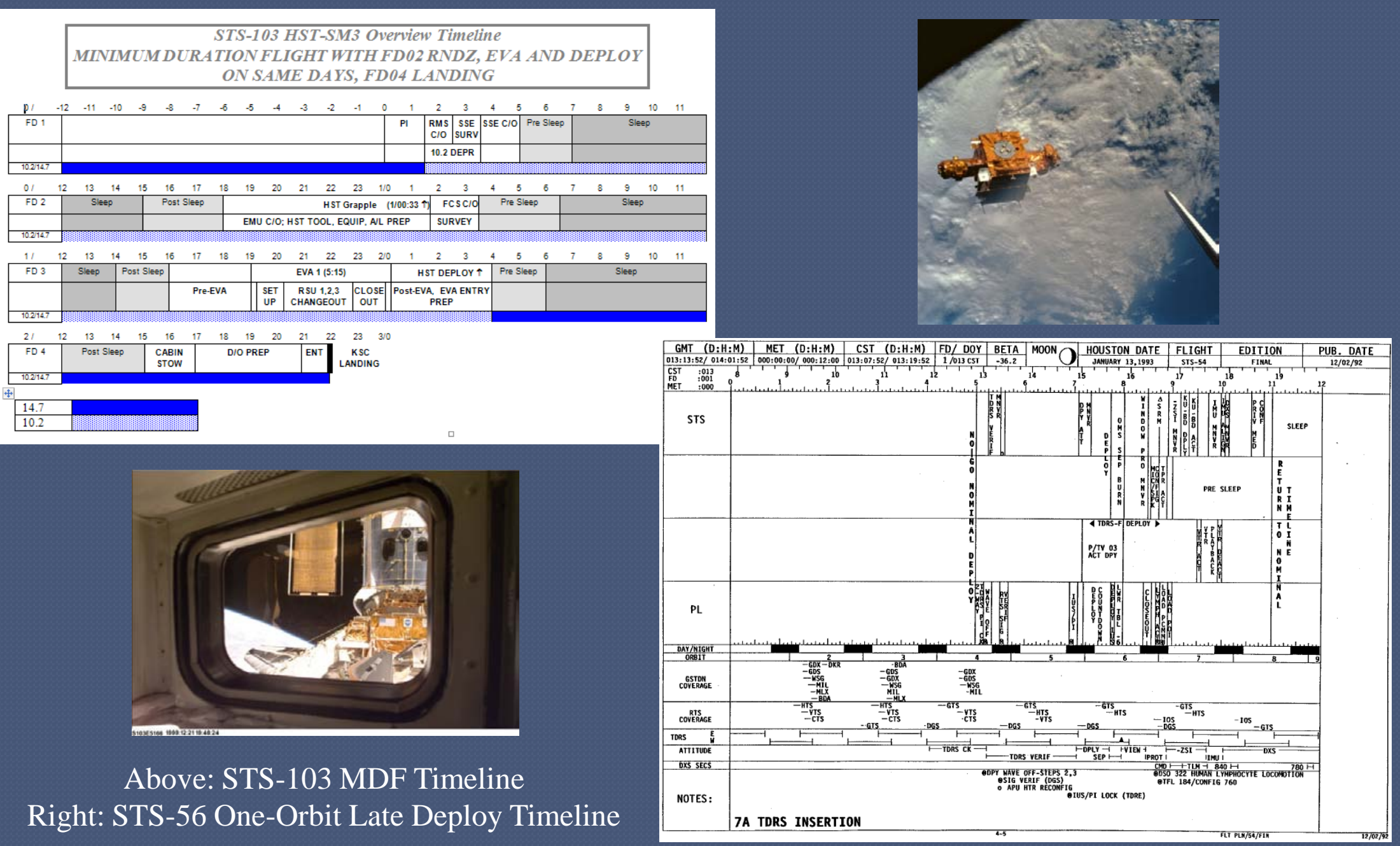




\section{Contingency and Alternate Timelines Lessons}

- Contingency planning can be time consuming, and the number of potential failures unending

- Every minute spent preflight paves the way for a faster turnaround time in-flight

o Focus should remain on those failures that are most likely to occur, or have the largest impact to mission success

- Flexibility in the plan is key, as planned responses can be utilized for similar, unexpected situations

- Train the contingency cases in simulations - keeps the team calm under pressure and organized when the response has been practiced 


\section{Planning Tools}

- Internal software applications to create the Flight Plans evolved with the increasing complexity of missions and improvements in technology

- Crew Activity Planning System (CAPS)

Manual entry of all activities, procedure references, durations

Scheduled on crew and graphically displayed in a "WYSIWYG" format, then printed out for crew and ground use during the mission

- Flight Planning System (FPS)

Allowed timelines created at MSFC with Spacelab tasks to be imported into the database to lessen the amount of manual data entry

Allowed information to be extracted from the timeline to sort and filter activities for analysis

- Consolidated Planning System (CPS)

Merged the capabilities of FPS with a system being developed for the ISS

Unfortunately, CPS project management was placed outside of the planning organization, which caused the software to go through several updates before it was practical for use In addition, data extraction was no longer available, and in-house scripts and macros had to be created to perform this task 


\section{Examples of Timelines}

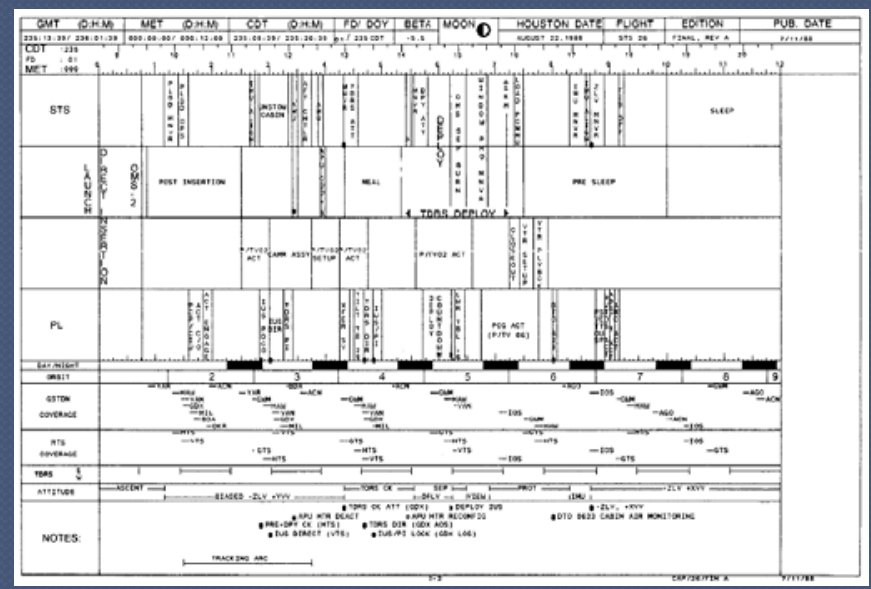

STS-26: CAPS Summary Timeline

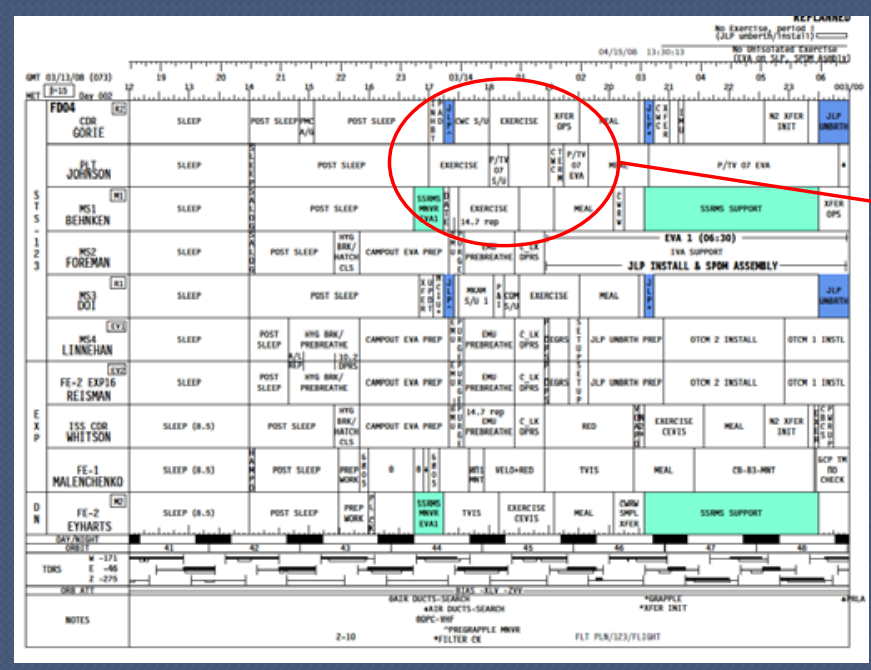

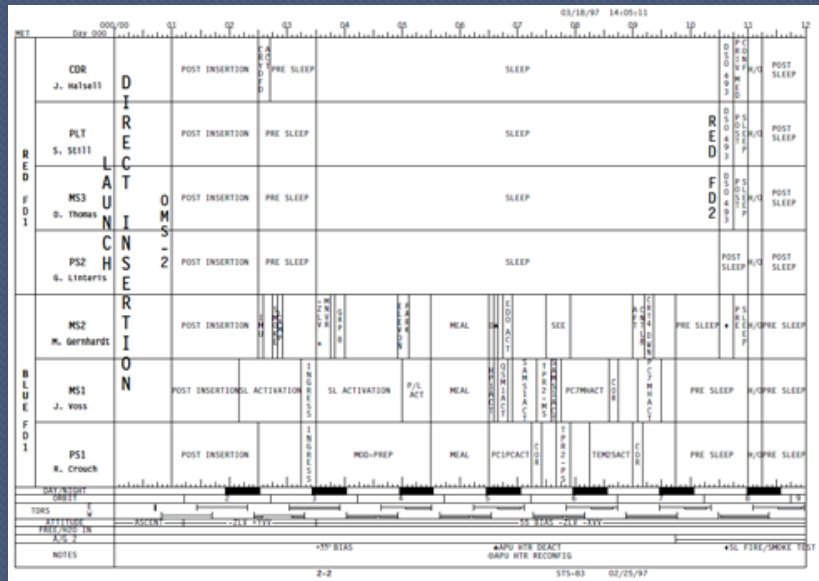

STS-83: FPS Summary Timeline

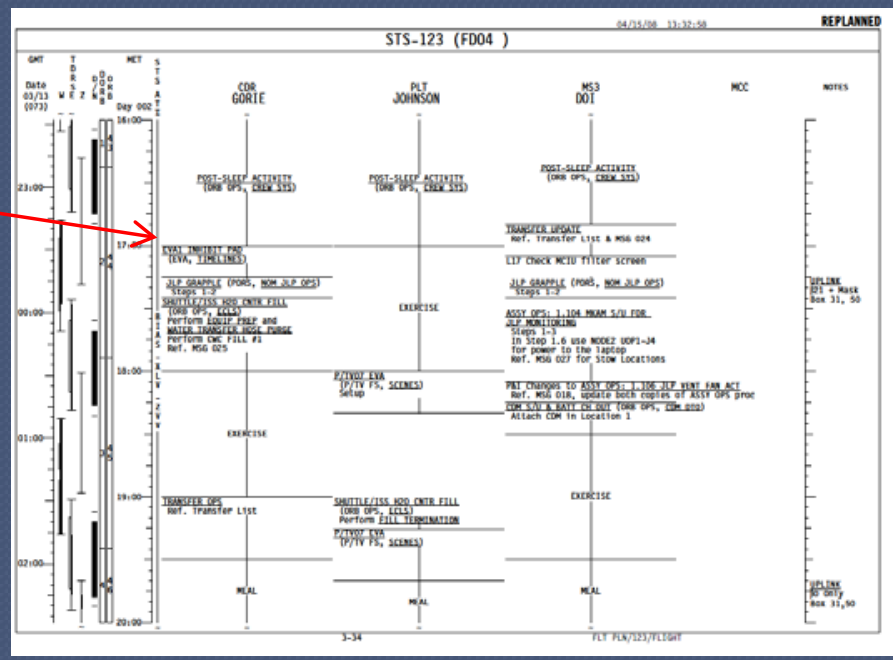

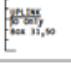

STS-123: CPS Summary and Detail Timelines 


\section{Planning Tools Lessons}

- Invest in software which has the capability to easily update timelines both preflight and real-time to establish and maintain the most efficient use of the crew's time

○ Include the users in the design system to ensure all requirements and preferences are addressed

- Focus should be placed on user interface

Too much focus on the functionality only will result in a tool that is not practical

- Project management should remain close to the users to allow for more frequent and efficient dialog to provide a useful application from the beginning

- Allow flexibility for the development of user scripts to augment the capabilities of the tool 


\section{Spacecraft Versatility and Flight Planning}

- The FAOs and Attitude and Pointing Officers were able to utilize the flexibility and versatility of the Orbiter to meet mission objectives, increase communication, and uplink and downlink crew data, while adhering to thermal requirements for external hardware

- Thermal Capabilities

The thermal envelope of the Orbiter was expanded over the course of the program to meet operational needs of the Space Shuttle, to solve unexpected problems, and to test the limits

- Mission Payloads and Mission Objectives

Payload requirements and even new mission requirements provided integration challenges that were solved by changes to the Orbiter's attitude

- Communication Line-of-Sight

Communication between the Shuttle and ground became increasingly utilized with the introduction of a high data rate antenna

Changes to the Orbiter's orientation were made to maximize comm coverage during critical time periods of the mission

- Data Uplink and Downlink

Use of the comm satellites allowed updates to procedures and Flight Plans to be uplinked to the crew From a teleprinter that printed a subset of ASCII characters to the Orbital Communications Adapter (OCA) that allowed access to the onboard laptops for file uplink, the increases in technology and available bandwidth dramatically improved the type of information transferred to and from the Shuttle 


\section{Examples of Attitude Timeline and Pointing Products}

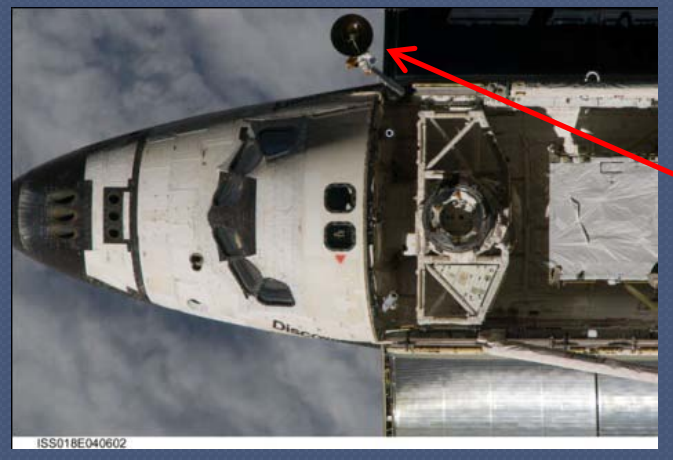

Ku-band Antenna

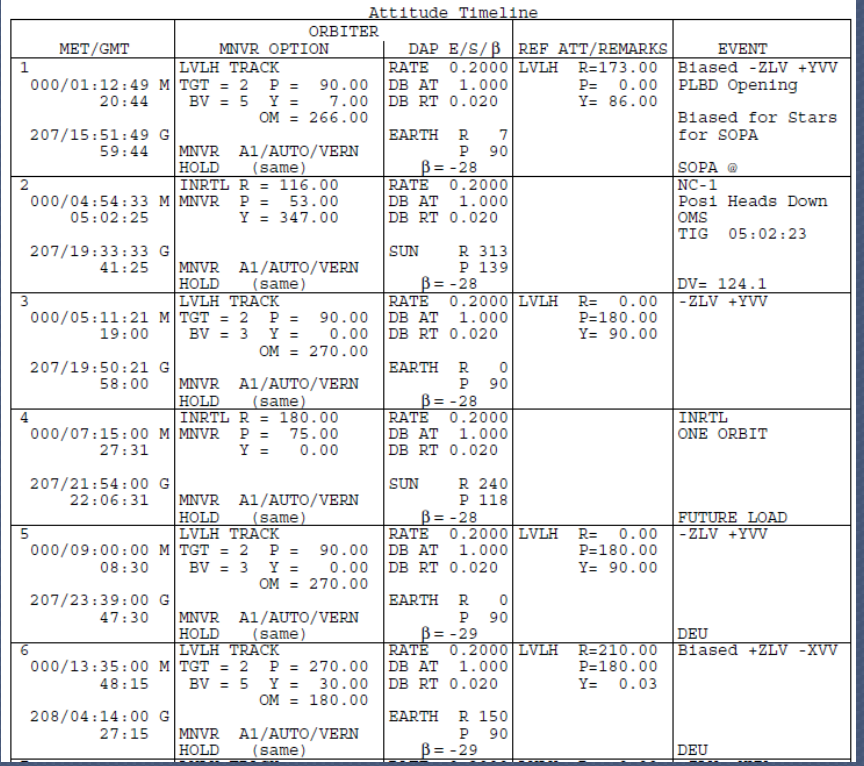

STS-114 Attitude Timeline

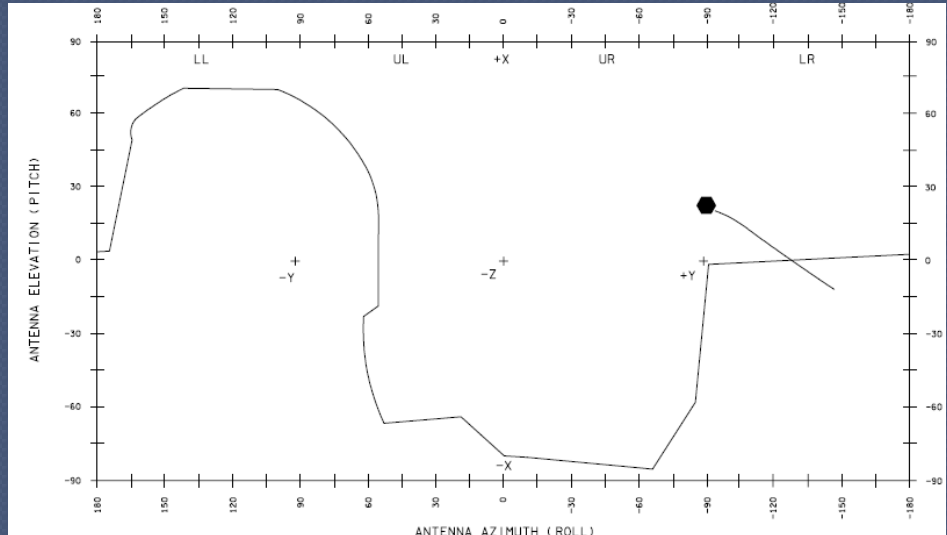

Sample Ku-band Plot

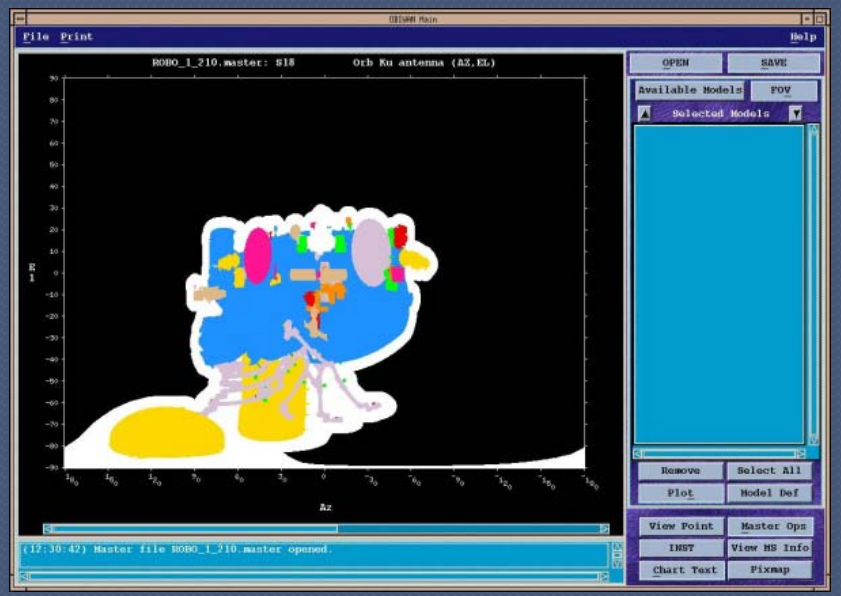

STS-135 MPLM Position Plot 


\section{Computers and Crew Message Uplinks}

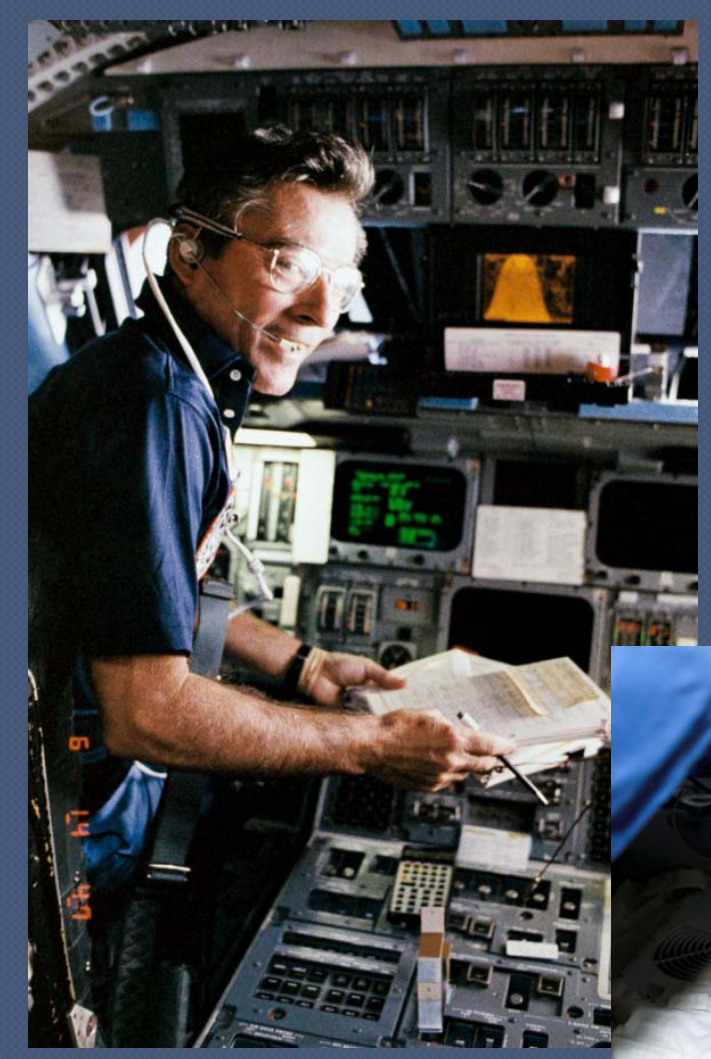

Above: STS-9, John Young in front of GRiD 1139 Compass laptop Right: STS-114, A31p laptops

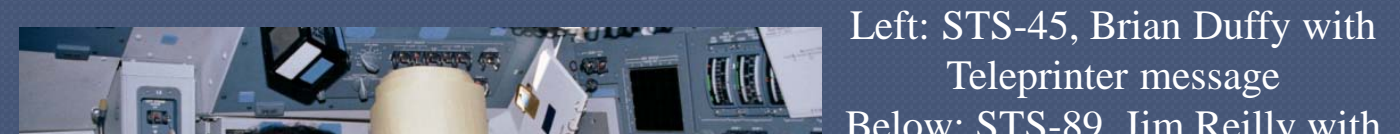
Teleprinter message TIPS printout

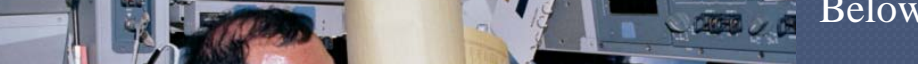

(x)
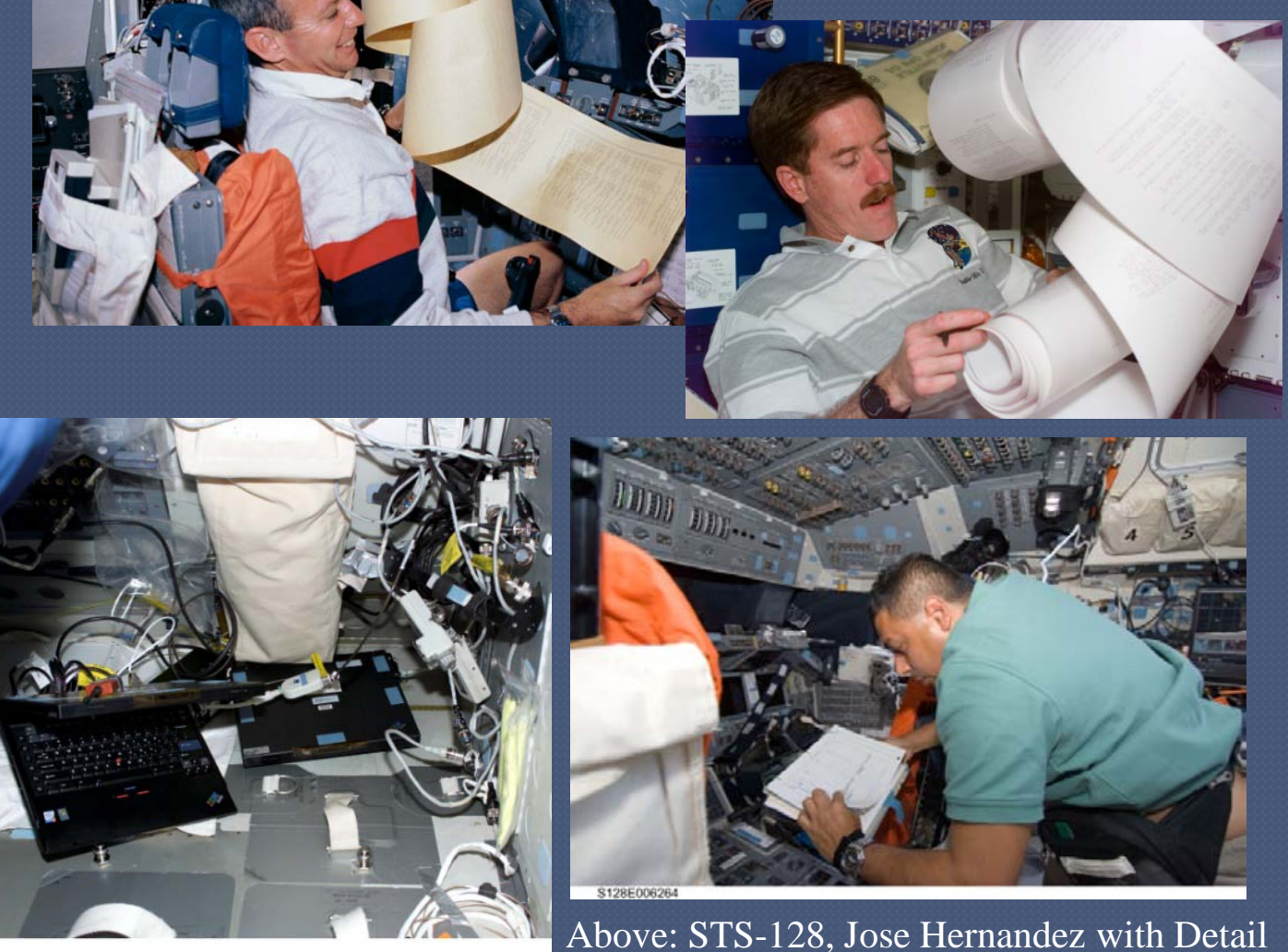

Above: STS-128, Jose Hernandez with Detail Timeline uplinked and printed via OCA 


\section{Spacecraft Versatility and Flight Planning Lessons}

- The ability to adapt is an important key

o Strive to provide as much operational flexibility in spacecraft orientation as possible to offset costs of analyzing a full operational envelope

o Explore all options with regard to communication, from tracking capability of antennae to changing the orientation of the spacecraft

- Anticipate spacecraft body and payload blockage, and have a means to protect hardware

- Allow room for upgrades to hardware and software to improve communication and transfer of ground and crew data, and provide a high data rate uplink system to allow large files to be sent to the crew 


\section{Conclusion}

- The success of future human spaceflight depends on how much preparation is completed prior to launch of the next program

- Space Shuttle missions provide a large amount of data concerning how astronauts live and operate in space, how ground controllers interface with the crew and each other, and how the versatility of the Orbiter itself provided the capability to perform a variety of missions

- Preflight planning, cooperative relationships, anticipation of contingencies pave the way for success

- Highly functional and user friendly tools for ground and on-orbit operations, and an adaptable spacecraft are also extremely important

- Whether to an asteroid, the Moon, Mars, or deeper into the Solar System, it is imperative that future mission planners learn from history, and stand on the shoulders of those before them 